\title{
Ectopic POU5F1 in the male germ lineage disrupts differentiation and spermatogenesis in mice
}

\author{
Yu Zheng, LeAnna J Phillips, Rachel Hartman, Junhui An and Christina T Dann \\ Department of Chemistry, Indiana University, Bloomington, Indiana, USA
}

Correspondence should be addressed to C T Dann; Email: ctdann@indiana.edu

\begin{abstract}
Expression levels of the pluripotency determinant, POU5F1, are tightly regulated to ensure appropriate differentiation during early embryogenesis. POU5F1 is also present in the spermatogonial stem cell/progenitor cell population in mice and it is downregulated as spermatogenesis progresses. To test if POU5F1 downregulation is required for SSCs to differentiate, we produced transgenic mice that ubiquitously express POU5F1 in Cre-expressing lineages. Using a Vasa-Cre driver to produce ectopic POU5F1 in all postnatal germ cells, we found that POU5F1 downregulation was necessary for spermatogonial expansion during the first wave of spermatogenesis and for the production of differentiated spermatogonia capable of undergoing meiosis. In contrast, undifferentiated spermatogonia were maintained throughout adulthood, consistent with a normal presence of POU5F1 in these cells. The results suggest that POU5F1 downregulation in differentiating spermatogonia is a necessary step for the progression of spermatogenesis. Further, the creation of a transgenic mouse model for conditional ectopic expression of POU5F1 may be a useful resource for studies of POU5F1 in other cell lineages, during tumorogenesis and cell fate reprogramming.

Reproduction (2016) 152 363-377
\end{abstract}

\section{Introduction}

The generations are connected by an undulating cycle of developmental potency. Mammalian embryonic development starts with a zygote that is totipotent, continues with the formation of a pluripotent blastocyst and culminates with the formation of the germ lineage, which eventually produces unipotent gametes that may unite to repeat the cycle. Much of this cycle of developmental potency is influenced by the transcription factor POU5F1 (OCT4) (Scholer et al. 1990b). In the absence of POU5F1, homozygous null mutant zygotes undergo normal cleavage cell divisions, but then they fail to form a pluripotent inner cell mass (ICM) at the blastocyst stage and die (Nichols et al. 1998). While the cells in the ICM are initially viable, their normally pluripotent state is altered to a differentiated trophoblast fate. Since the ICM is the source of pluripotent embryonic stem cells, ES cells cannot be derived from Pou5f1 mutants. Furthermore, by manipulating POU5F1 levels in established ES cells, differentiation and loss of pluripotency occur following only twofold alterations to POU5F1 levels (Niwa et al. 2000).

POU5F1 also functions later in embryonic development in primordial germ cells (PGCs). To overcome the peri-implantation lethality of Pou $5 f 1$ knockout mice, Pou5f1 flox mice were generated
(Kehler et al. 2004). A Cre driver was used to delete Pou5f1 in PGCs mid-gestation. In contrast to its function in the early embryo, in PGCs, the absence of POU5F1 led to apoptosis and impaired fertility. While some PGCs persisted, and in adult males some mature spermatozoa were even formed, the presence of the floxed allele in spermatozoa suggested that the remaining germ cells were the result of incomplete Cre excision (Kehler et al. 2004).

POU5F1 function was also examined in postnatal male germ cells in vitro (Dann et al. 2008, Wu et al. 2010). These experiments utilized RNA interference to knockdown POU5F1 in cultures containing spermatogonial stem cells (SSCs). One study transiently introduced small-interfering RNA into cells, but no effect on SSC self-renewal was seen (Wu et al. 2010). Another group used transgene-derived small hairpin RNA and showed a requirement for POU5F1 in proliferation and viability (Dann et al. 2008). They also showed that cultured SSCs with POU5F1 knockdown had reduced stem cell colonization ability following transplantation, suggesting a role for POU5F1 in SSC self-renewal. However, understanding the function of POU5F1 in the postnatal germ lineage in a true in vivo context remains unknown. 
The expression pattern of POU5F1 throughout development parallels its function in maintenance of pluripotency and the germ lineage. In the early embryo, it is initially expressed equally in all blastomeres. Then, its expression in the outer cells decreases, as those cells form the trophectoderm and POU5F1 becomes restricted to the ICM by the blastocyst stage (Rosner et al. 1990, Scholer et al. 1990a, Palmieri et al. 1994). It is initially highly expressed throughout the ICM, but then becomes restricted once again during mid-gestation and disappears from all cells except the PGCs (Rosner et al. 1990, Pesce et al. 1998). POU5F1 is initially strongly expressed in the PGCs of the embryo and its expression is downregulated before the onset of meiosis in both sexes (Pesce et al. 1998). In females, downregulation of POU5F1 mRNA and protein occurs at embryonic day 14.5 (Pesce et al. 1998). In males, however, meiosis begins postnatally and is preceded by a complex series of proliferation, migration and differentiation events. The timing of POU5F1 downregulation in the male germ lineage is not clear from the published reports and clarifying the timing of POU5F1 downregulation is relevant to understanding its role during these male-specific events.

During late embryogenesis in males, PGCs undergo a transient mitotic arrest before forming gonocytes, the term for male germ cells present at birth in rodents. A few days after birth, gonocytes exit mitotic quiescence, migrate from the lumen to the basal membrane of the seminiferous tubules and then a subset of cells initiates the first wave of spermatogenesis, while another subset generates the first pool of SSCs. Steady-state adult spermatogenesis depends on the self-renewal of SSCs, but the first wave of meiotic cells is thought to originate directly from differentiated spermatogonia, not SSCs (Yoshida et al. 2006). Subsequent waves of spermatogenesis originate from SSCs that proliferate and form transit amplifying progenitor cells and ultimately fully differentiated spermatogonia. The SSCs and transit amplifying progenitor cells are collectively called 'undifferentiated spermatogonia,' and following further proliferation and the onset of KIT expression, the cells are called 'differentiated spermatogonia' (SchransStassen et al. 1999). Ultimately, the differentiated spermatogonia undergo meiosis and spermiogenesis, forming spermatozoa.

There is consensus that POU5F1 protein is present in gonocytes and in undifferentiated spermatogonia (Pesce et al. 1998, Tadokoro et al. 2002, Filipponi et al. 2007). POU5F1 may be limited to only a small subset of the undifferentiated spermatogonia known as $A_{s}$ and $A_{p r}\left(A_{\text {single }}\right.$ and $\left.A_{\text {pair }}\right)$, some of which include the true SSCs (Tadokoro et al. 2002, Filipponi et al. 2007). However, a few contradictory reports have shown that POU5F1 is present in a broader group of spermatogonia that includes $A_{a l} \quad\left(A_{\text {aligned }}\right)$ and differentiated spermatogonia (Kehler et al. 2004, Hofmann et al. 2005,
Tokuda et al. 2007, Ketkar \& Reddy 2012). Nonetheless, POU5F1 has often been described as a marker for SSCs.

Pou5f1 gene expression in mice has also been studied by analyzing expression of reporter transgenes. The well-studied GOF18 PE transgene contains $18 \mathrm{~kb}$ of genomic DNA with GFP (including stop codon and SV40 polyadenylation site) inserted at the start codon of Pou5f1, and with an epiblast-specific 986 bp 'proximal enhancer' removed (Yeom et al. 1996). Multiple laboratories have made transgenic mice with GOF18 $\Delta$ PE, and one line ('OG2') is publicly available and widely utilized (Szabo et al. 2002). There is consensus that GOF18 $\mathrm{PE}$ (herein, 'Oct4-GFP') mice express GFP in PGCs and neonatal spermatogonia, but only some have seen GFP in adult spermatogonia (Ohbo et al. 2003, Garcia \& Hofmann 2012, Azizi et al. 2016). Interestingly, Oct4-GFP expression has been reported in KIT+ differentiated cells and in long chains of spermatogonia, suggesting that GFP from the transgene may be more widely expressed than endogenous POU5F1 (Ohbo et al. 2003).

The goal of our study was to clarify the expression pattern of OCT4 during spermatogenesis in pups vs adults, and to determine the relevance of POU5F1 regulation for neonatal and adult spermatogenesis. We found that POU5F1 is widely expressed in undifferentiated spermatogonia in pups and continues to be present in rare undifferentiated spermatogonia of the adult. By creating transgenic mice with ectopic POU5F1 expression that persists in the germ lineage, we found that POU5F1 downregulation is important for spermatogonia to progress through differentiation into meiosis during the first wave of spermatogenesis. Interestingly, undifferentiated spermatogonia were maintained throughout adulthood and sometimes produced a complete round of spermatogenesis, suggesting that gonocytes transitioned to establish an initial pool of functional stem cells that were subsequently maintained through self-renewal. However, the majority of tubules in adults lacked differentiated cells, similar to the situation during the first wave, demonstrating the importance of POU5F1 downregulation for spermatogenesis in pup and adult stages.

\section{Materials and methods}

\section{Mice}

All procedures involving mice were approved by the Bloomington Institutional Animal Care and Use Committee. cOct4 transgenic mice were generated and maintained on a pure $\mathrm{C} 57 \mathrm{BL} / 6$ background. cOct4 mice are available from The Jackson Laboratory as Stock No. 029084. Oct4-GFP (B6;CBA$\operatorname{Tg}($ Pou5f1-EGFP)2Mnn/J), Stra8-Cre (Tg(Stra8-icre)1Reb/J) and Vasa-Cre (FVB-Tg(Ddx4-cre)1Dcas/) breeder pairs were from The Jackson Laboratory and maintained by breeding with wild-type mice of equivalent background. Certain indicated 
experiments used Oct4-GFP heterozygotes, which were produced by breeding with $\mathrm{C} 3 \mathrm{H}$ or $\mathrm{C} 57 \mathrm{Bl} 6$.

For fertility testing, three cOct4; Vasa-Cre (from lines 53 and 18) and three control (WT or Vasa-Cre) 2-3-month-old males were paired with wild-type females. Control males were paired with one female for 3 weeks and each sired two litters. cOct4; Vasa-Cre males were paired with females for 2.5-6.5 weeks and were checked daily for the presence of a vaginal plug. Each cOct4; Vasa-Cre male was kept in a breeding test until at least two plugs were noted for each of at least two females. No litters were sired by any of the cOct4; Vasa-Cre males tested for the duration of the study.

\section{GS cell culture and proteosome inhibitor treatment}

GS cells (cell line DGC3) from a DBA/2 mouse were derived and cultured as described (Heim et al. 2011). MG132 (Calbiochem) was reconstituted in ethanol at $10.5 \mathrm{mM}$ and stored at $-80^{\circ} \mathrm{C}$ for no more than 1 month. MG132 was diluted in culture medium just before use and treatment times ranged from 0 to $4 \mathrm{~h}$ as indicated.

\section{Immunostaining of whole tubules}

Whole tubule immunostaining was performed as published with modifications (Gassei \& Orwig 2013). Testes or separated seminiferous tubules were rocked in $4 \%(\mathrm{v} / \mathrm{v})$ paraformaldehyde or $10 \%(\mathrm{v} / \mathrm{v})$ neutral buffered formalin at $4{ }^{\circ} \mathrm{C}$ overnight. Tubules were washed with PBS (phosphate-buffered saline) for $15 \mathrm{~min}$ twice, treated with mild dehydration solution (PBS with $10 \%$ (v/v) methanol and $0.1 \%(\mathrm{v} / \mathrm{v})$ TritonX-100) at $4^{\circ} \mathrm{C}$ for $1 \mathrm{~h}$ and blocked with PBSMT (PBS with 1xRoche blocking solution (Roche) and $0.5 \%(\mathrm{v} / \mathrm{v})$ TritonX-100) at $4{ }^{\circ} \mathrm{C}$ overnight. Tubules were incubated with primary antibody diluted in PMSMT (rabbit anti-POU5F1 1:100, mouse anti-GFP 1:500, rabbit anti-SALL4 1:800 or goat anti-GFRA1 1:50) at $4{ }^{\circ} \mathrm{C}$ overnight. Antibody details are in Supplementary Table 1, see section on supplementary data given at the end of this article. Tubules were washed in PBT (PBS with $0.1 \%(\mathrm{v} / \mathrm{v}$ ) TritonX-100) six times for $15 \mathrm{~min}$. Secondary antibodies were diluted in PBT with $1 \mu \mathrm{g} / \mathrm{mL}$ DAPI and applied for $1 \mathrm{~h}$ followed by six $15 \mathrm{~min}$ washes in PBT. Tubules were mounted on glass slides in Vectashield or Fluoro-Gel mounting medium. Images were obtained using a Nikon NiE upright microscope equipped with a Hamamatsu Orca-Flash 2.8 sCMOS camera or with a Nikon Eclipse TiS inverted microscope equipped with a Retiga 2000R Fast 1394 camera. Images were acquired with Q-capture Pro software, and Image J was used for pseudocoloring and to create overlays of colors.

\section{Immunostaining of sectioned testes}

Testes were dissected and fixed in $4 \%(\mathrm{v} / \mathrm{v})$ paraformaldehyde for cryosections or in formalin for histology at $4{ }^{\circ} \mathrm{C}$ for $2 \mathrm{~h}$ to overnight (depending on tissue size). Sections stained with hematoxylin/eosin were prepared from tissue embedded in paraffin using standard procedures. For immunostaining, tissue was saturated with increasing concentrations of sucrose and embedded in O.C.T. Compound (Fisher Healthcare,
Houston, TX, USA). Ten micron sections were cut and stored at $-80^{\circ} \mathrm{C}$ before staining. Cryosections were rehydrated with PBS, blocked for $15 \mathrm{~min}$ in $0.1 \mathrm{M}$ glycine, permeabilized in PBT for $5 \mathrm{~min}$ and blocked for $30 \mathrm{~min}$ in $1 \%(\mathrm{w} / \mathrm{v})$ bovine serum albumin (Jackson ImmunoResearch) diluted in PBT. For GFRA1 immunostaining, PBT was replaced with PBS, and for GFRA1/STRA8 costaining, permeabilization was with PBS containing $10 \%(\mathrm{v} / \mathrm{v})$ methanol. Primary antibodies and dilutions used were: rabbit anti-DAZL (1:1000), rabbit antiPOU5F1 (1:200), rabbit anti-SALL4 (1:500), rabbit anti-STRA8 $(1: 200)$, rabbit anticleaved caspase $3(1: 500)$, rabbit anti-CREM (1:200), rat anti-Tra98 (1:500), goat anti-GFRA1 (1:50) or mouse anti-GFP (1:800). Antibody details are in Supplementary Table 1. Primary antibodies were applied overnight at $4^{\circ} \mathrm{C}$. Appropriate secondary antibodies conjugated with Alexa594, Alexa-488 or Cy3 were diluted (1:500) in PBT (or PBS for GFRA1 staining) with $3.6 \mathrm{e}-6 \mathrm{~mol} / \mathrm{L}$ DAPI and applied for $1 \mathrm{~h}$ at room temperature or $4^{\circ} \mathrm{C}$ overnight. Secondary antibodies were from Jackson ImmunoResearch, Life Technologies and Cell Signaling Technology. Where not specified, incubations/ washes were at room temperature. For a negative control, the primary antibody was omitted. Images were obtained as described above.

\section{cOct4 transgene construction}

The cOct4 plasmid was derived from pLLU2G (Addgene \#21620, Cambridge, MA, USA), which contains the human Ubiquitin $C$ (Ubc) promoter that we and others have shown previously to be active in a variety of cell types including germ cells (Schorpp et al. 1996, Dann et al. 2006). We removed sequences of pLLU2G that were irrelevant to this study by digestion, Klenow fill-in and relegation (Smal/Xbal to remove $\mathrm{U} 6$ and LoxP and EcoRI/Pvull to remove LoxP and WPRE). A T2A/furin ribosomal skip sequence was amplified with TD462/TD498 primers (Supplementary Table 2) and inserted by traditional molecular cloning 5' of eGFP (Yang et al. 2008, Fanslow et al. 2014). The Pou5f1 coding sequence was amplified from mouse ES cell cDNA and sequence verified upon insertion into a unique Agel site $5^{\prime}$ to T2a/furin. A $\mathrm{BGH}$ polyA-addition site was PCR amplified from pCDNA4/myc-His (Invitrogen) with primers TD598/TD599, and isothermal ligation was used to insert the sequence 3' of eGFP (Gibson et al. 2010a,b). The LoxP-flanked stop cassette, which contained stop codons and a triplicate repeat of SV40 polyA-addition sites, was amplified from Ai2 plasmid (Addgene \#22796) using TD595/TD596 primers and was cloned into a Nhel site immediately 3' of the Ubc promoter by traditional molecular cloning (Madisen et al. 2010). Enzymes for plasmid construction were from New England Biolabs (Ipswich, MA, USA), and primers synthesized at Integrated DNA Technologies (Coralville, IA, USA). Critical regions of the plasmid including Pou5f1, T2A, eGFP and LoxP sites were confirmed by Sanger sequencing. The plasmid design was validated by cotransfecting HEK293 cells with the indicated plasmids using Lipofectamine2000 following manufacturer's guidelines (Thermo Fisher) and lysing cells 3 days after transfection. Ngn3-Cre (DM\#259) was from Addgene and Ubc-Cre was constructed in our lab (Gu et al. 2002). 


\section{cOct4 transgenic mouse production and genotyping}

The cOct4 transgene fragment was prepared for injection by $\mathrm{Accl} /$ Pvull digestion and gel purification. The linearized transgene was injected into pronuclei of C57BL/6 embryos using standard procedures at the UT Southwestern Medical Center Transgenic Core facility (Dallas, TX, USA). Fifty-five candidate mice were screened by PCR and 11 founders were obtained, five of which could be bred and transmitted the transgene to the next generation. Genotyping was performed on ear, tail or neonatal toe-derived DNA using standard PCR and gel analysis procedures. Alternatively, the quick-release reagent from Phire Animal Tissue Direct PCR kit (Thermo Fisher) in combination with quantitative PCR analysis with Maxima Sybr (Thermo Fisher) on a 480 LightCycler (Roche) was employed for routine genotyping, using wild-type genomic DNA as a comparison. Supplementary Table 2 lists the primers used, and primers for Stra8-Cre and Vasa-Cre genotyping were described previously (Gallardo et al. 2007, Sadate-Ngatchou et al. 2008). Transnetyx genotyping service was also used.

For PCR analysis of genomic DNA from cauda epididymalderived swimming spermatozoa, DNA extraction was performed using a published method (Griffin 2013). In brief, lysis was conducted by adding extraction buffer $(4.2 \mathrm{M}$ guanidine thiocyanate, $100 \mathrm{mM} \mathrm{NaCl}, 1 \%$ Sarkosyl, $150 \mathrm{mM}$ DTT and $200 \mu \mathrm{g} / \mathrm{mL}$ Proteinase $\mathrm{K}$ ) and incubating at $56^{\circ} \mathrm{C}$ for $2 \mathrm{~h}$ and then the DNA was precipitated with isopropanol and rehydrated. A transgene-specific primer pair (TD535/YZ2) that flanked the floxed stop cassette was used to detect the unexcised vs excised versions of the transgene by amplification using Herculase II polymerase (Agilent), and products were analyzed with gel electrophoresis.

\section{Cell dissociation and sorting}

Testes were dissected and rinsed in DMEM/F-12 medium, the tunica albuginea was removed and seminiferous tubules were separated with forceps. After rinsing twice in DMEM/F-12, tubules were digested in $1 \mathrm{mg} / \mathrm{mL}$ Collagenase Type IV (Sigma) with $7 \mathrm{mg} / \mathrm{mL}$ DNase for $15 \mathrm{~min}$ at $37^{\circ} \mathrm{C}$. After washing twice in Hanks' balanced salt solution, tubules were incubated in $0.25 \%$ trypsin/EDTA with $7 \mathrm{mg} / \mathrm{mL}$ DNase I (Sigma) for $20 \mathrm{~min}$ at $37^{\circ} \mathrm{C}$. The digestion was stopped by adding approximately one-tenth volume fetal bovine serum (FBS; Hyclone, Logan, UT, USA) and mixing vigorously. Cell samples were strained with a 40 micron strainer and then spun and resuspended in PBS with 2\% (w/v) FBS. Dissociated cells were incubated for 25 min with antibodies or isotype controls diluted in PBS with $2 \%(w / v)$ FBS. Supplementary Table 1 lists antibodies used. Sorting was performed on an Aria II (BD Bioscience, San Jose, CA, USA).

\section{Immunoblotting}

Cells or tissue were washed in PBS before lysis. For sorted cells, equal numbers $(50,000-100,000)$ of cells were resuspended in sample buffer ('SB,' Laemmli buffer with $0.1 \mathrm{M}$ DTT) and boiled at $100^{\circ} \mathrm{C}$ for $5 \mathrm{~min}$. Testes were lysed in SB using a FastPrep-24 instrument (MP Biochemicals, Santa Ana, CA, USA) with Matrix D beads for $20-60 \mathrm{~s}$ at $4 \mathrm{~m} / \mathrm{s}$.
Amounts of beads and SB were scaled according to the weight of tissue lysed. Lysates were electrophoresed through a $10 \%$ SDS-PAGE gel and transferred onto PVDF membrane using standard procedures. Following blocking in 5\% milk with PTw (PBS+10\% Tween 20), the membrane was incubated in mouse anti-POU5F1 (1:250), washed in PTw, incubated in goat antimouse IgG-HRP secondary and washed in PTw. Supersignal West Femto (Thermo Fisher) was used for detection. Blots were stripped using standard procedures and reprobed with anti-tubulin to verify even loading.

\section{Statistical analysis}

Mean and standard deviation or standard error mean, as indicated, were calculated with sample sizes described in figure legends. The $t$-test function in Excel (Microsoft) was used to calculate $P$-values based on assumptions of unpaired data with equal variance.

\section{Results}

\section{POU5F1 expression in pup and adult spermatogonia}

While it is known that POU5F1 is expressed in undifferentiated spermatogonia, to our knowledge, a direct quantitative comparison of expression during neonatal and adult spermatogenesis has not been reported. Using Oct4-GFP mice, we sorted undifferentiated spermatogonia (THY1+/GFP+) and differentiated spermatogonia (THY1-/GFP+). The equivalent cell populations from pup (dpp 13) and adult testes were compared. We found that POU5F1 could only be detected in the THY1+ population of cells (Fig. 1A and B) and that POU5F1 levels within this sorted cell population were lower in adult than in pups (Fig. 1B). Consistent with the immunoblotting, by immunostaining whole seminiferous tubules, we observed dim nuclear staining of endogenous POU5F1 in cells that coexpressed Oct4-GFP (Fig. 1D-K). Endogenous POU5F1 could be detected in both pups and adults, but the POU5F1 + cells were very rare in adults (Fig. 1H-K). POU5F1 was seen in individual, paired and sometimes chains of spermatogonia. Similar results were obtained by immunostaining sections of tubules (Fig. 1L-S). For unknown reasons, POU5F1+ cells were more readily detected in the Oct4-GFP reporter mouse strain compared with other strains (data not shown). Our results confirmed published data showing that POU5F1 is present in undifferentiated spermatogonia, and is typically present in a small subset of $A_{s}$ and $A_{p r}$ undifferentiated spermatogonia. Furthermore, the rarity of detectable POU5F1 in adults suggested that the fraction of spermatogonia with POU5F1 is lower in adults compared with pups.

Immunoblotting and immunostaining approaches both showed that the Oct4-GFP reporter was expressed in a broader group of cells including long chains of spermatogonia where endogenous POU5F1 could 
A
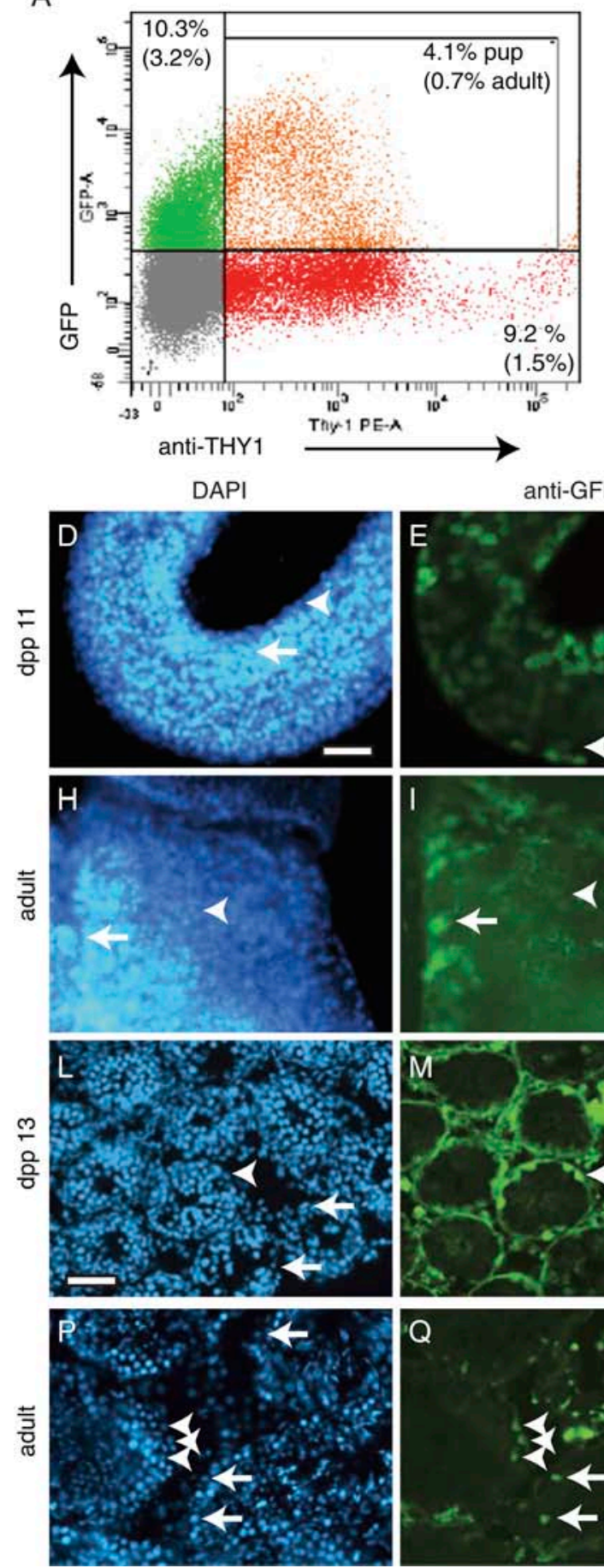

B

C

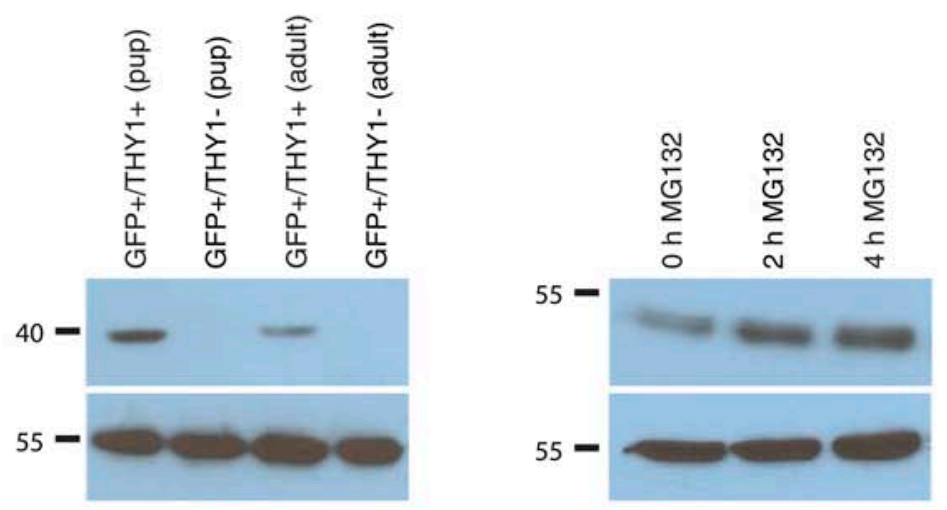

anti-GFP
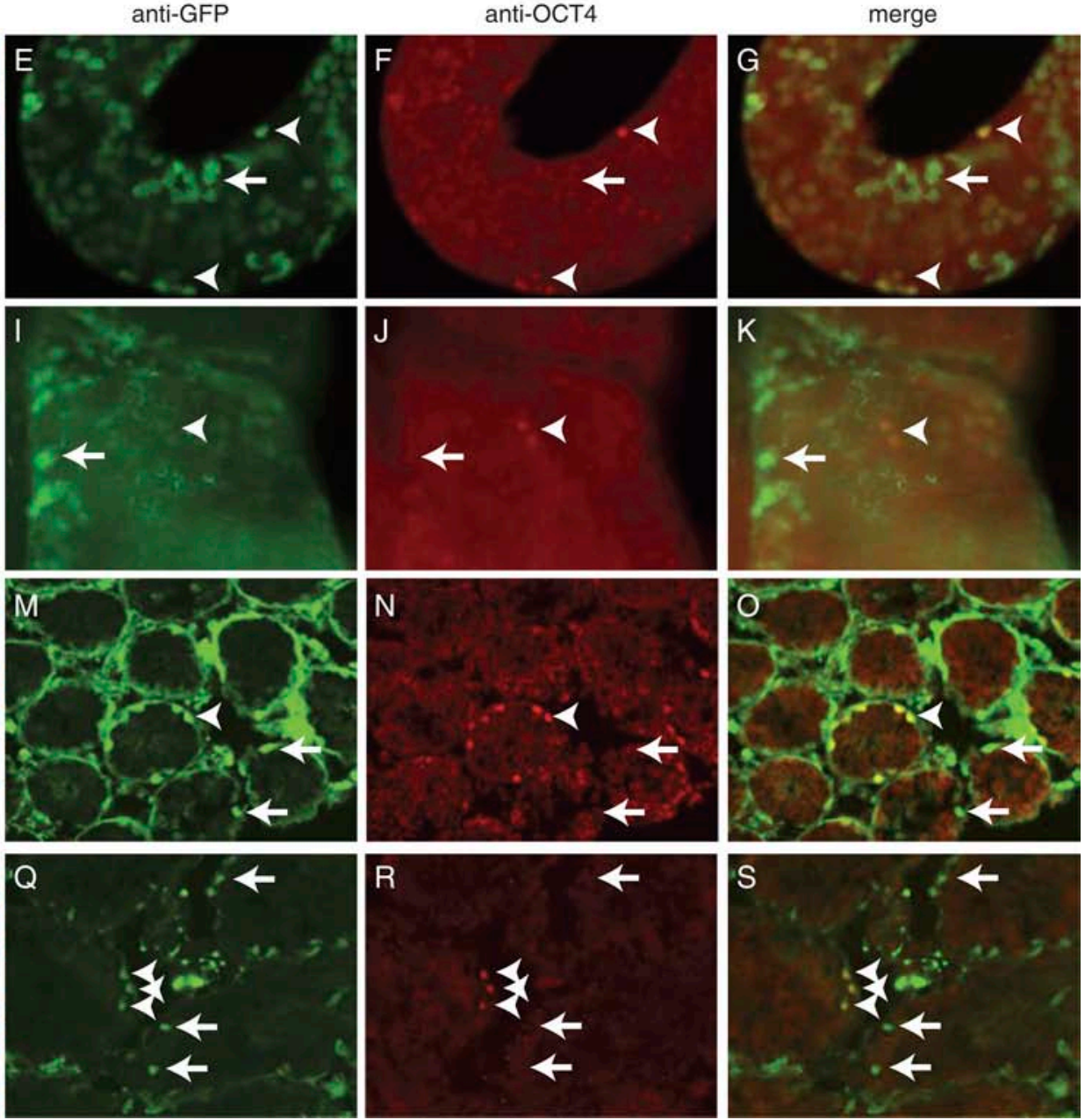

Figure 1 POU5F1 protein expression in Oct4-GFP pup and adult testes. (A) Dot plot showing cells sorted from heterozygous Oct4-GFP pup (dpp 13) testes and the percentage of parent gate cells for each subpopulation. Parent gates (not shown) include forward and side scatter to select singleton, viable cells. Percentages of the corresponding populations from adult Oct4-GFP mouse are shown in parenthesis. Isotype controls were used to define background fluorescence for the CD90 (THY1) antibody (not shown). (B) POU5F1 (top) or TUBULIN (bottom) immunoblotting of indicated sorted cell fractions. (C) POU5F1 (top) or TUBULIN (bottom) immunoblotting of cultured spermatogonial stem/ progenitor GS cells treated for 0,2 or $4 \mathrm{~h}$ with $30 \mu \mathrm{M}$ MG132. Size standards (kDa) are indicated. (D-S) Immunostaining of whole tubules (D-K) or cryosections (L-S) from dpp $11(\mathrm{D}-\mathrm{G})$, dpp 13 (L-O) or adult (H-K and P-S) Oct4-GFP heterozygous mice. (D, H, L and P) DAPI (DNA), (E, I, $\mathrm{M}$ and Q) GFP immunostaining, ( $\mathrm{F}, \mathrm{J}, \mathrm{N}$ and $\mathrm{R})$ POU5F1 immunostaining or (G, K, O and S) merge of GFP and POU5F1. Arrows point to cells with GFP and without endogenous POU5F1 and arrowheads point to cells with both GFP and POU5F1. Bar, $50 \mu \mathrm{m}$. 
not be detected. One possible explanation was that POU5F1 protein is regulated by posttranslational mechanisms, while the GFP reporter reflects only Pou5f1 promoter activity but not posttranscriptional/ translational regulation. To test whether POU5F1 may be regulated by proteosome-mediated degradation, we used cultured spermatogonia ('GS' cells), which have been shown previously to express POU5F1 heterogeneously, likely reflecting the mixed population of SSCs and partly differentiated transit amplification populations that are present (Kanatsu-Shinohara et al. 2003, Dann et al. 2008). Treatment with the proteosome inhibitor MG132 led to stabilization of POU5F1, indicating that POU5F1 is subject to downregulation by the proteosome and consistent with the possibility that it may be directly regulated by the destabilizing modification, ubiquitin (Fig. 1C).

\section{Generation of transgenic mice for conditional ectopic expression of POU5F1}

Given the known importance of POU5F1 levels in controlling cell fate during early embryogenesis, we hypothesized that downregulation of POU5F1 could be important for the progression of spermatogenesis.

A cOct4
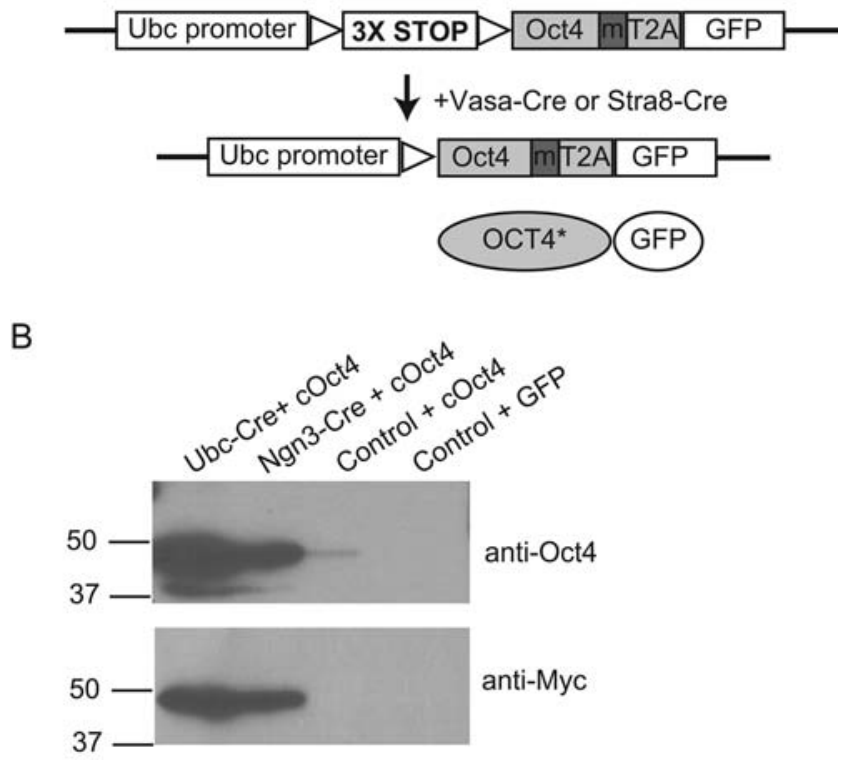

Figure 2 cOct4 transgene design and validation in cultured cells. (A) cOCT4 transgene with Ubiquitin $\mathrm{C}$ promoter, LoxP sites (triangles), $3 \times$ repeated SV40 polyA stop cassette, Pou5f1 coding sequence, Myc tag ('m'), T2A ribosomal skip sequence and GFP coding sequence. Following excision by Cre, transcription and translation, it is expected that the POU5F1 protein contains a c-terminal Myc epitope tag and T2A peptide ('POU5F1*') while GFP is unmodified. (B) POU5F1 (top) or MYC (bottom) immunoblotting of HEK293 cells transfected with the indicated plasmids. Size standards $(\mathrm{kDa})$ are indicated.
To test this idea, we created a transgene ('cOct4') designed to ubiquitously express POU5F1 and GFP following Cre-mediated excision of a stop cassette flanked by LoxP sites (Fig. 2A). Transient transfections of the transgene with and without Cre recombinase into cultured cells validated that the transgene produced exogenous POU5F1 and GFP in the presence of Cre (Fig. 2B and data not shown).

Transgenic mice were obtained by DNA injection into pronuclei of $\mathrm{C} 57 \mathrm{BL} / 6$ embryos, and several transgenic lines were established for analysis. We initially used a Stra8-Cre driver, which was reported to produce Cre in postnatal spermatogonia and spermatocytes (SadateNgatchou et al. 2008). Testes from cOct4;Stra8-Cre mice and sibling controls were analyzed from 1- and 2-month-old mice. Transgene-derived POU5F1 could be detected by immunoblotting of testicular lysates from multiple lines (Fig. 3A and B); occasionally, transgene expression was also observed in cOct4 mice without Stra8-Cre, albeit at lower levels than the doubly transgenic mice, suggesting that there was a low level of expression that presumably occurred by read-through
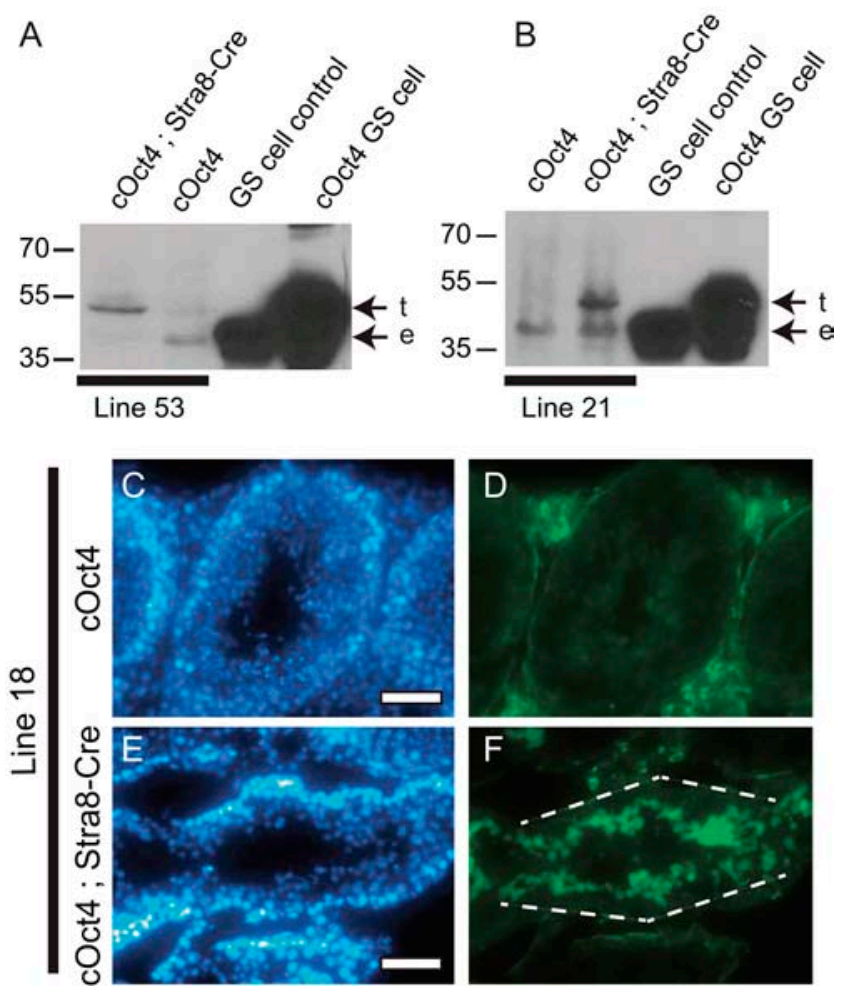

Figure 3 Transgene expression in cOct4;Stra8-Cre mice. (A and B) POU5F1 immunoblotting of testicular lysates from dpp 31 cOct 4 or cOct4;Stra8-Cre mice of line 53 (A) or dpp 66 mice of line 21 (B). Cultured spermatogonial GS cells from wild-type mice or from mice containing cOct4 were used as a comparison. Size standards (kDa) are indicated and the arrows point to endogenous (' $\mathrm{e}$ ') and transgenic (' $\mathrm{t}$ ') bands. (C-F) Immunostaining to detect GFP in testes from cOct4 and cOct4;Stra8-Cre dpp 31 mice of line 18. DAPI (DNA) staining (C and E), GFP immunostaining (D and F). Bar, $50 \mu \mathrm{m}$. 
of the floxed stop cassette (Supplementary Fig. 1 and data not shown). Surprisingly, we did not observe any abnormalities in seminiferous tubule cross sections in cOct4;Stra8-Cre mice (Fig. 3E and data not shown). One possible explanation was that transgene (GFP) expression appeared to be limited to a small subset of tubules, and it was only visible in haploid spermatids but not in spermatogonia (Fig. 3F). Altogether, our analysis of the cOct4;Stra8-Cre mice showed that Cre excision activated the transgene and produced ectopic POU5F1 in testes, but excision may have been inefficient and occurred in later spermatogenic stages than expected. Evidently, the amount of ectopic POU5F1 that was produced was insufficient to lead to a phenotype and/or the presence of POU5F1 in postmeiotic germ cells was inconsequential (see 'Discussion' section).

\section{cOct4; Vasa-Cre mice have a severe disruption in spermatogenesis}

Next, we used a Vasa-Cre driver to examine the effect of ectopic POU5F1 during neonatal spermatogenesis. VasaCre male mice were reported to produce Cre in the germ lineage starting during late embryogenesis in gonocytes (Gallardo et al. 2007). In contrast to cOct4;Stra8-Cre mice, the cOct4;Vasa-Cre mice exhibited a distinctive defect in spermatogenesis. The testes of adult mice were visibly smaller in cOct4;Vasa-Cre mice for two independent lines (descendants from founders 53 and 18; Fig. 4A and Supplementary Fig. 2). Histological examination of seminiferous tubule cross sections from adult mice showed a dramatic and heterogeneous defect. Many seminiferous tubules contained only spermatogonia and Sertoli cells (Fig. 4E and Supplementary Fig. 2). Occasional tubules contained the complete progression of germ cells from spermatogonia to spermatozoa and others contained no germ cells (Supplementary Fig. 2). Interestingly, the phenotype of seminiferous tubules from a third line of mice (descendants of founder 21) had qualitatively the same array of defects as in line 53 and line 18; however, quantitatively, the phenotype was far less severe with most tubules containing apparently normal spermatogenesis (Supplementary Fig. 2). Analysis of epididymal cross sections revealed similar heterogeneity, with almost no spermatozoa evident in the caput of cOct4; Vasa-Cre mice from lines 18 or 53, and a higher frequency of caput cross sections containing spermatozoa in mice from line 21 (Fig. 4F and Supplementary Fig. 2). Male cOct4;Vasa-Cre mice from lines 18 and 53 were subjected to breeding tests and failed to sire any progeny (Supplementary Fig. 2).

Immunoblotting confirmed the presence of transgenederived POU5F1 in testicular lysates (Fig. 4B); it should be noted that the samples used in the immunoblotting experiment were total testicular lysates that differed widely in their composition of germ cell numbers and cell types, precluding conclusions about relative levels
A

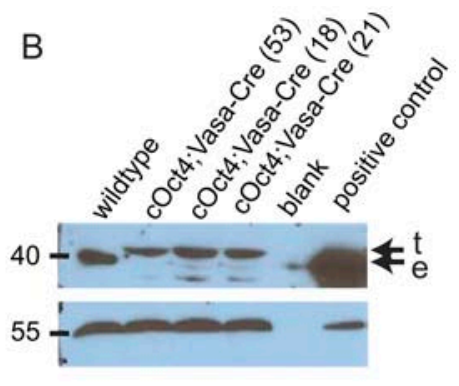

Testes
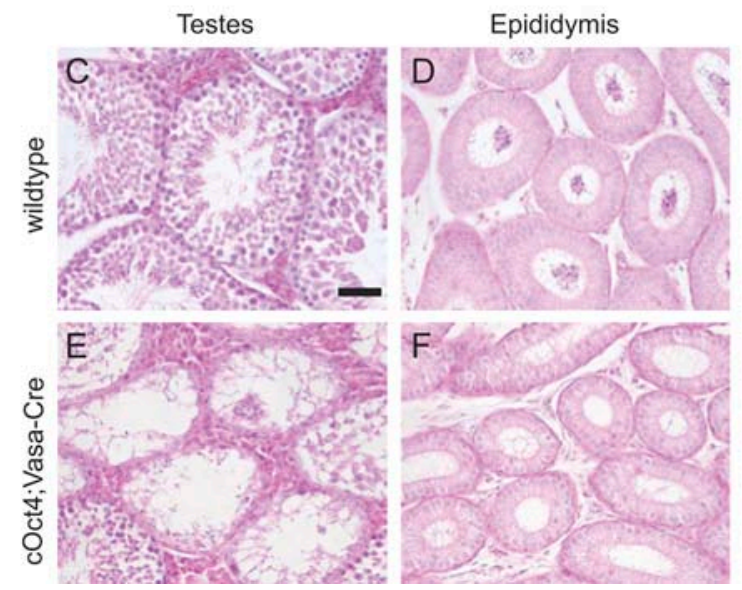

Figure 4 Phenotypic analysis of adult cOct4; Vasa-Cre testes. (A) Testes of 2-month-old cOct4;Vasa-Cre mice (line 53 and 18) and sibling controls. (B) POU5F1 (top) and TUBULIN (bottom)

immunoblotting of testicular lysates from 5 -month-old mice of lines

53, 18 and 21 cOct4; Vasa-Cre and wild type. ES cells were used as a positive control. Size standards $(\mathrm{kDa})$ are indicated and the arrows point to endogenous ('e') and transgenic ('t') bands. (C-F) Hematoxylin/eosin staining of wild type (C and D) and cOct4; Vasa-Cre ( $\mathrm{E}$ and F) 2-month testes ( $\mathrm{C}$ and $\mathrm{E}$ ) and caput epididymis (D and F). Bar, $50 \mu \mathrm{m}$.

between the samples. PCR analysis of spermatozoa recovered from the caput epididymis of cOct4; Vasa-Cre mice from line 21 confirmed the presence of the Creexcised version of the transgene (Supplementary Fig. 3). We chose to use lines 18 and 53 for additional analysis because these lines had the most severe germ cell loss phenotype.

\section{SSCs self-renew to sustain spermatogenesis in the long term in cOct4; Vasa-Cre mice, but germ cell differentiation is disrupted}

Immunostaining was used to further define the extent of germ cell loss in adult cOct4; Vasa-Cre mice. TRA98 was used to detect all germ cells, SALL4 and GFRA1 to detect undifferentiated spermatogonia and CREMtau to detect meiotic cells (Foulkes et al. 1992, Tanaka et al. 1997, Gassei \& Orwig 2013). Most tubule cross sections contained at least one TRA98-positive cell and the pattern and number of germ cells present were variable. Interestingly, we frequently $(\sim 35 \%$ of 509 tubules in 13 mice aged 2-12 months) saw 

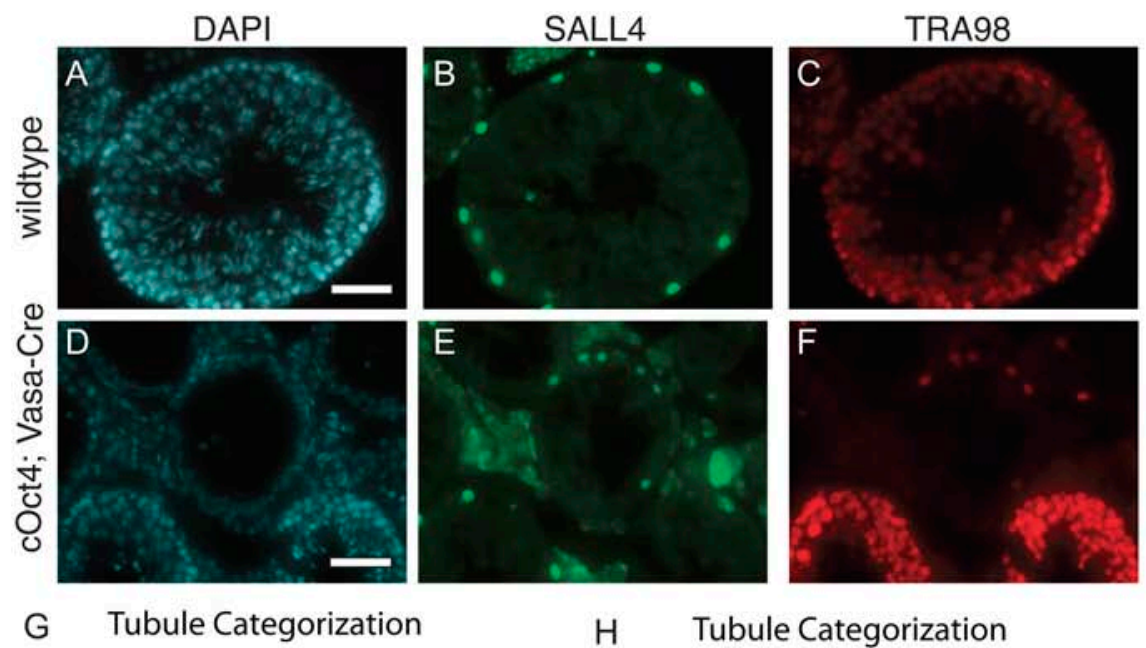

H Tubule Categorization
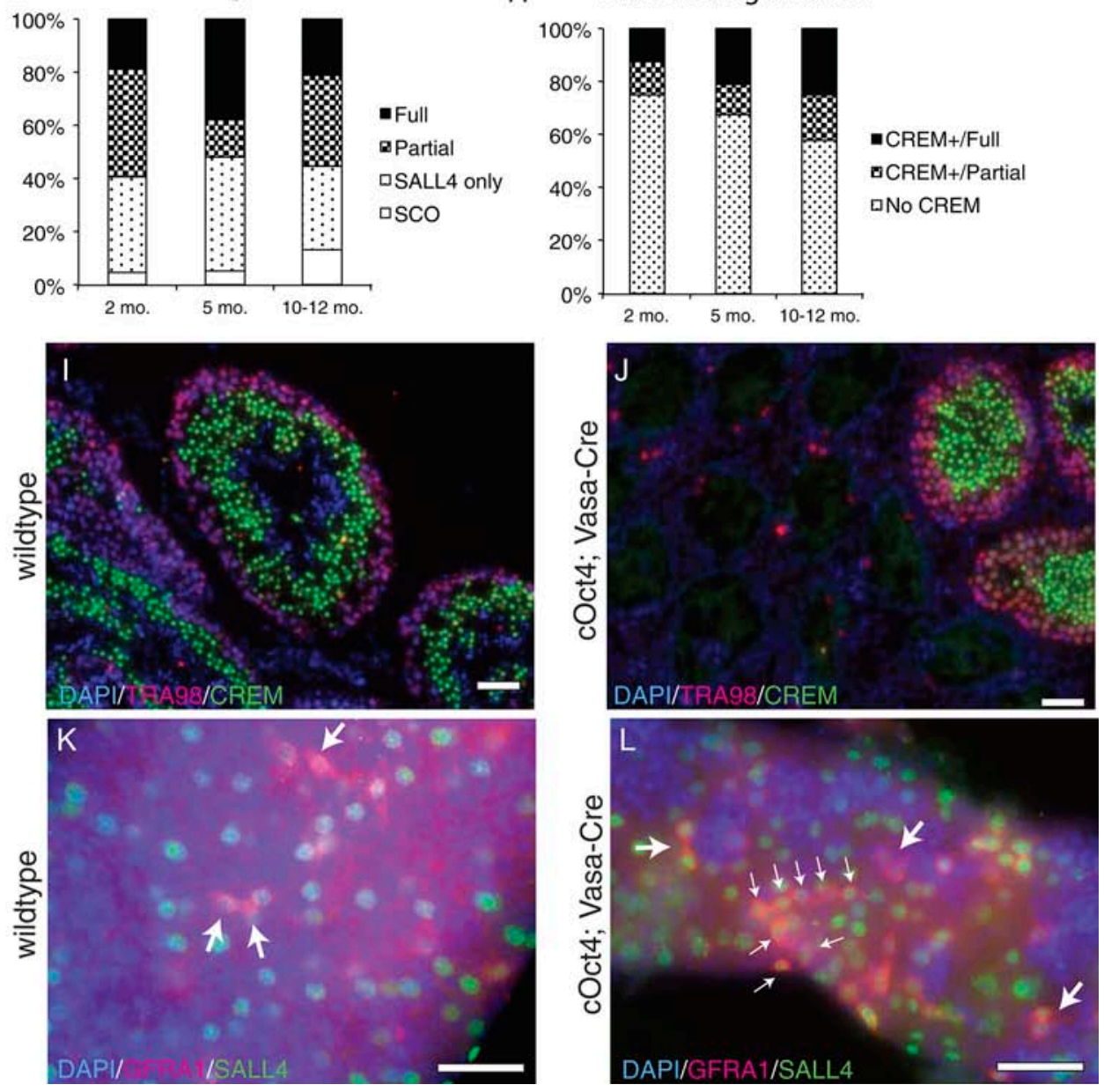

Figure 5 Immunostaining of adult cOct4; Vasa-Cre testes. (A, B, C, D, E and F) SALL4 (green) and TRA98 (red) coimmunostaining to detect undifferentiated spermatogonia in wild-type (A-C) and cOct4; Vasa-Cre (D-F) 2-month mice. (G) Mean percentage of tubule cross sections with no germ cells (SCO), only SALL4+ germ cells ('SALL4+ only'), partial germ cell recovery and full germ cell recovery in 2 month ( $n=6$ mice), 5 month ( $n=3$ mice) and 10-12-month ( $n=4$ mice) cOct4; Vasa-Cre. 240, 109 and 160 tubules were scored for 2, 5 and 10-12 months respectively. (H) Mean percentage of tubule cross sections with no CREM staining ('No CREM'), some CREM staining but abnormal pattern ('CREM+/Partial') or CREM staining in a normal pattern including nuclei/DAPI with morphology of elongating spermatids ('CREM+/Full'). 1652, 387 and 548 tubules were scored for 2, 5 and 10-12-month mice respectively. (I and J) CREM (haploid spermatids) and TRA98 coimmunostaining in cross sections from wild-type (I) and cOct4; Vasa-Cre (J) 2-month mice. Images shown are a merge of CREM (green), TRA98 (red) and DAPI (blue). (K and L) SALL4 and GFRA1 co-immunostaining in whole seminiferous tubules from wild-type (K) and cOct4; Vasa-Cre (L) 2-month mice. Images shown are a merge of SALL4 (green), GFRA1 (red) and DAPI (blue). Arrows point to GFRA1+ spermatogonia. Bar, $50 \mu \mathrm{m}$. 
tubule cross sections where $100 \%$ of the TRA98 cells costained with SALL4. Therefore, in these tubules, undifferentiated spermatogonia were the sole remaining germ cell type (Fig. 5E-G). Consistent with the idea that a block occurred during spermatogonial differentiation, most tubules $(\sim 70 \%$ of 2587 tubules in 13 mice aged 2-12 months) also lacked CREM-tau staining (Fig. 5H).

Based on SALL4 and TRA98 immunostaining, we categorized the patterns visible in tubule cross sections as having no TRA98+ germ cells ('Sertoli cell only' or SCO), only SALL4+/TRA98+ germ cells ('SALL4 only'), partially recovered spermatogenesis (some TRA98+/ SALL4- cells, but in an abnormal pattern) or fully recovered spermatogenesis (normal pattern). Comparison of 2-month-old mice with older mice revealed similar frequencies of tubules with only SALL4+ germ cells (Fig. 5G). The continued presence of undifferentiated spermatogonia, including mice older than 10 months, suggested that SSC self-renewal occurred in cOct4;VasaCre. Further supporting this conclusion, GFRA1, another marker of undifferentiated spermatogonia and SSCs, persisted throughout adulthood (Figs 5L and 6C-F) (Hofmann et al. 2005, Nakagawa et al. 2010).

While the majority of tubules lacked meiotic cells, there was a significant number of tubules that appeared to contain normal spermatogenesis. The presence of some tubules with CREM+ cells and elongating spermatids ('CREM+/Full') may have been caused by insufficient
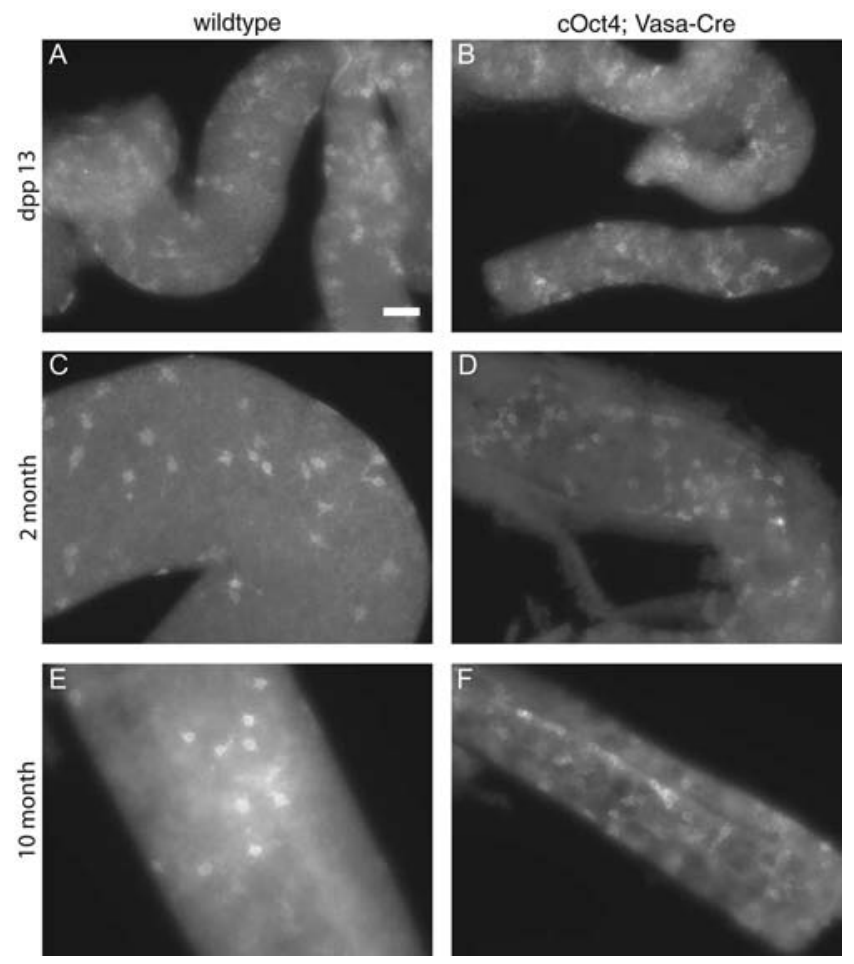

Figure 6 GFRA1 immunostaining in cOct4;Vasa-Cre. (A-F) GFRA1 immunostaining of whole seminiferous tubules from dpp 13 $(A$ and $B), 2$ month $(C$ and $D)$ and 10 month (E and $F$ ) in wild type (A, C and E) and cOct4; Vasa-Cre (B, D and F). Bar, $50 \mu \mathrm{m}$. overexpression of POU5F1, and/or overriding regulatory mechanisms that downregulate POU5F1 or work redundantly to block differentiation/spermatogenesis (Fig. $5 \mathrm{H}-\mathrm{J}$ ). Nonetheless, spermatogonial differentiation and germ cell maturation were greatly disrupted in the majority of tubules, showing that POU5F1 downregulation is an important step for the progression of spermatogenesis.

\section{The germ cell population begins to be reduced in neonates around dpp 4}

Given the severe lack of germ cells in adult cOct4; Vasa-Cre mice, we sought to identify the earliest point in spermatogenesis that was affected. Histological analysis revealed an overt phenotype beginning at dpp 13. While spermatocytes were evident in controls at dpp 13, cOct4;Vasa-Cre mice
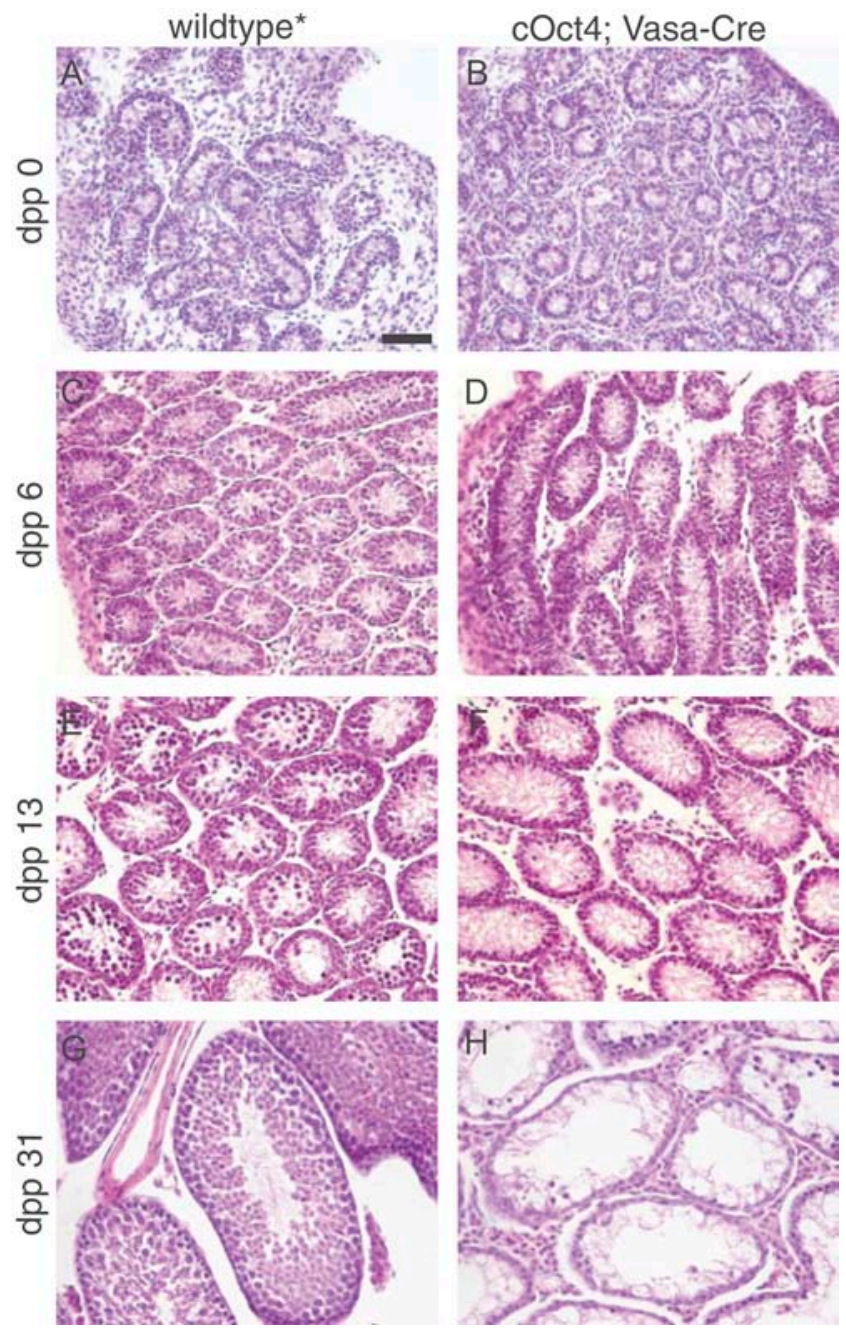

Figure 7 Phenotypic analysis of neonatal cOct4;Vasa-Cre testes. Hematoxylin/eosin staining of dpp 0 (A and B), dpp 6 (C and D), dpp 13 ( $E$ and $F$ ) and dpp 31 ( $G$ and $H)$ testes from control (A, C, E and $G$ ) and cOct4; Vasa-Cre (B, D, F and H) mice. Wild-type sibling controls are shown except in (C), which is a cOct4 sibling control. Bar, $50 \mu \mathrm{m}$. 

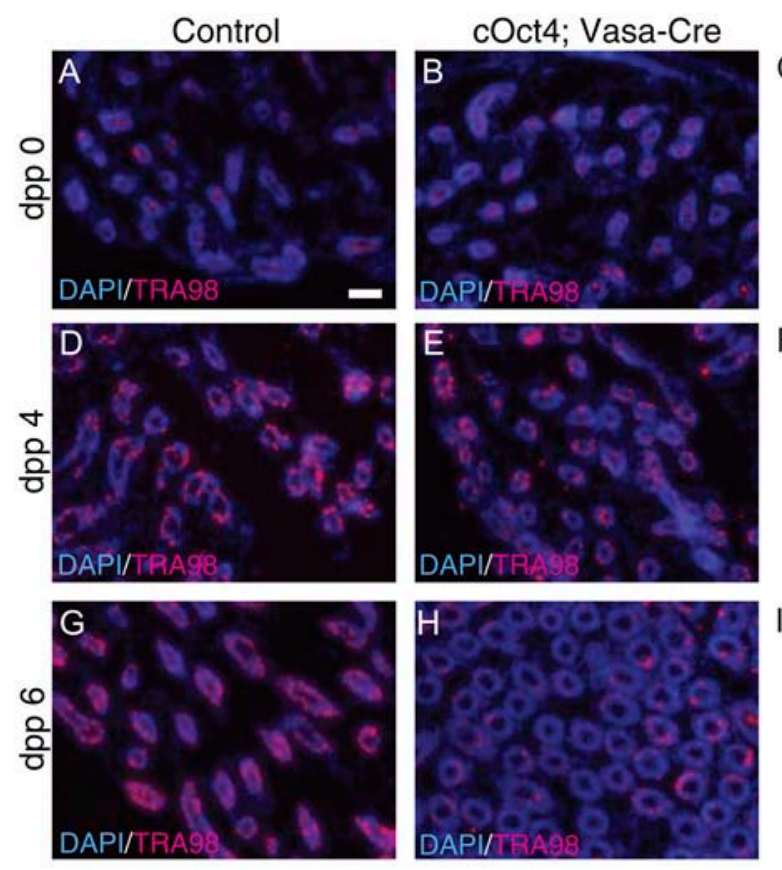
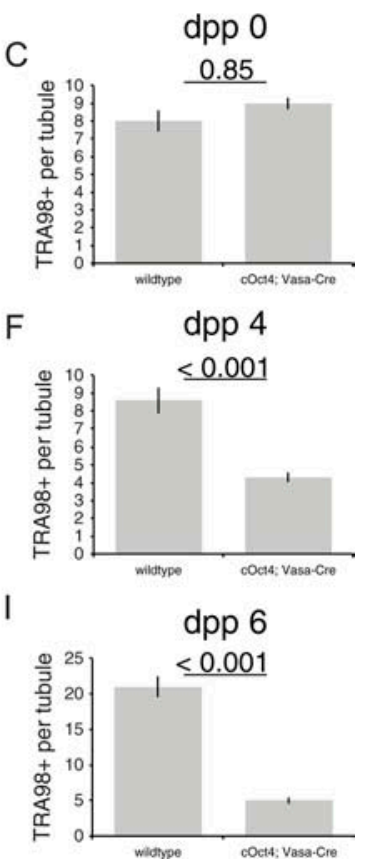

Figure 8 Germ cell loss in neonates of cOct4; Vasa-Cre. TRA98 immunostaining to detect germ cells in seminiferous tubule cross sections at dpp 0 (A and B), dpp 4 (D-F) and dpp $6(\mathrm{G}-\mathrm{I})$. Images shown are a merge of DAPI (blue) and TRA98 (red) and are from wild-type or Vasa-Cre controls (A, D and G) and cOct4; Vasa-Cre (B, E and $\mathrm{H}$ ) mice. (C, F and I) Histograms show the mean and standard error mean of the number of TRA98+ cells present per tubule cross section for 80 wild-type tubules (2 mice) and 73 cOct4; VasaCre tubules (2 mice) at dpp 0, for 74 wild-type tubules (2 mice) and 234 cOct4; Vasa-Cre tubules (5 mice) at dpp 4, and for 144 wild-type tubules (3 mice) and 320 cOct4; Vasa-Cre tubules (6 mice) at dpp 6. Quantitative analysis was restricted to circular (transverse) tubule cross sections. $P$-values are shown; Bar, $50 \mu \mathrm{m}$. had a scarcity of meiotic cells (Fig. 7E-F). Testes from dpp 6 appeared generally normal, with spermatogonia being present at the basal membrane as expected (Fig. 7C-D). However, following immunostaining with pan-germ cell markers, it became apparent that there were fewer germ cells in cOct4; Vasa-Cre mice at dpp 6 and dpp 4 (Fig. 8C-F and Supplementary Fig. 4). In contrast, the number of gonocytes at dpp 0 was similar in cOct4; Vasa-Cre compared with controls (Fig. 8A and B). Our analysis showed that the first point in postnatal development when downregulation of POU5F1 is critical is during the establishment of the first spermatogonia from gonocytes.

\section{Gonocytes undergo an abnormal transition to spermatogonia during the initiation of the first wave of spermatogenesis}

Several important events for the establishment of spermatogenesis occur during dpp 4 to dpp 6. Some gonocytes are transitioning to form the first pool of SSCs. Concurrently, other gonocytes are thought to directly differentiate. Recently, Niedenberger and coworkers proposed a model in which GFRA1 marks the first SSCs, while GFRA1-negative cells represent the differentiated spermatogonia that constitute the first wave of spermatogenesis (Niedenberger et al. 2015). Given the normal presence of endogenous POU5F1 in gonocytes and undifferentiated spermatogonia, we predicted that the SSC pool would be established normally despite the presence of cOCT4;Vasa-Cre. To assess whether the establishment of the first SSC pool was affected, we performed GFRA1 double staining with a pan-germ cell marker (TRA98 and SALL4 in neonates) to determine the fraction of total germ cells with GFRA1. By assessing the fraction, rather than the absolute number of GFRA1 + cells, we accounted for the observation that in cOct4; Vasa-Cre mice, the total germ cell population was reduced. Consistent with our prediction, while the total number of germ cells was smaller, the fraction that was GFRA1+ was not significantly different between cOct4;Vasa-Cre and GFRA1 + tended to even be higher in cOct4;VasaCre (Fig. 9A-G). Similar GFRA1+ staining results were obtained using whole tubule immunostaining approaches (Supplementary Fig. 4). Then, we tested whether the reduction in total germ cell number could be explained by a block in differentiation in cOct4; Vasa-Cre mice. Differentiation can be visualized by the onset of STRA8 expression (Zhou et al. 2008, Niedenberger et al. 2015). While STRA8+ cells were clearly less frequent in cOCT4; Vasa-Cre, and the fraction of STRA8+ germ cells tended to be lower, the difference was not significantly significant (Fig. 9H-N). In wild-type mice, GFRA1 and STRA8 immunostaining are generally expected to be in mutually exclusive subsets of spermatogonial populations - namely, the undifferentiated and differentiated spermatogonia respectively (Niedenberger et al. 2015). However, in cOct4; Vasa-Cre mice, at dpp 6, we frequently observed GFRA1+ cells that were also STRA8+ (Fig. 9O-U). The presence of STRA 8 and GFRA1 in the same cells likely indicates abnormalities in the differentiation program and may explain the inability to detect a clear alteration in the fractions of germ cells comprising the undifferentiated and differentiated spermatogonia. 


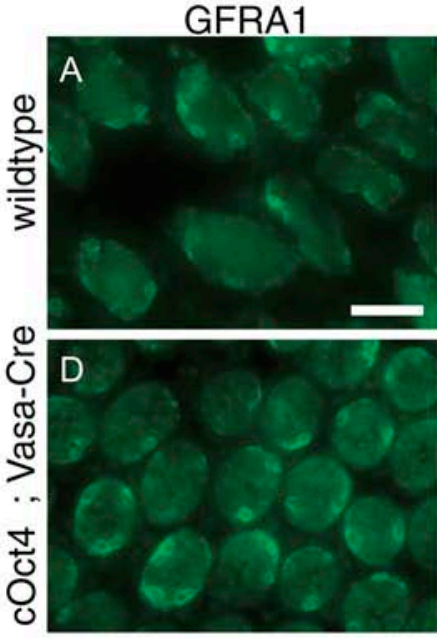

STRA8

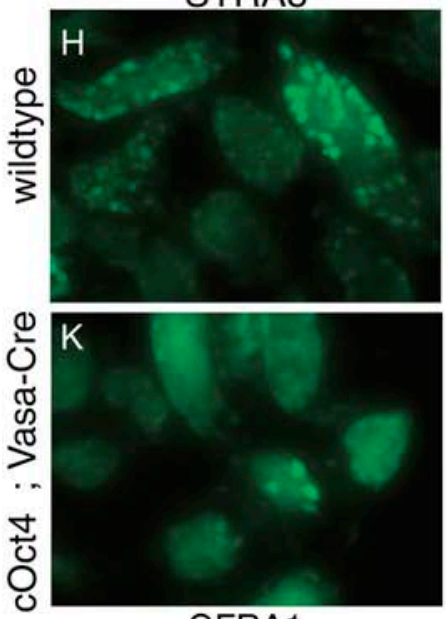

GFRA1
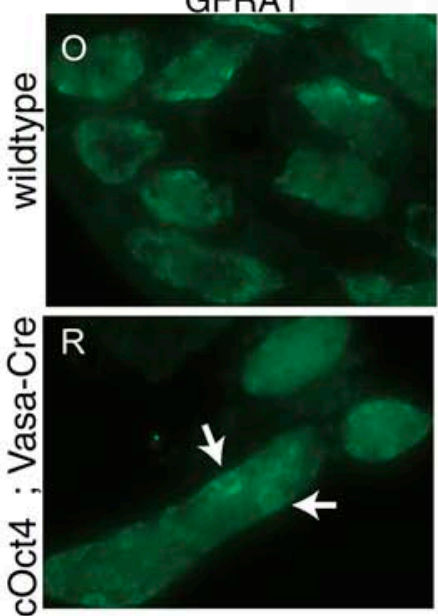
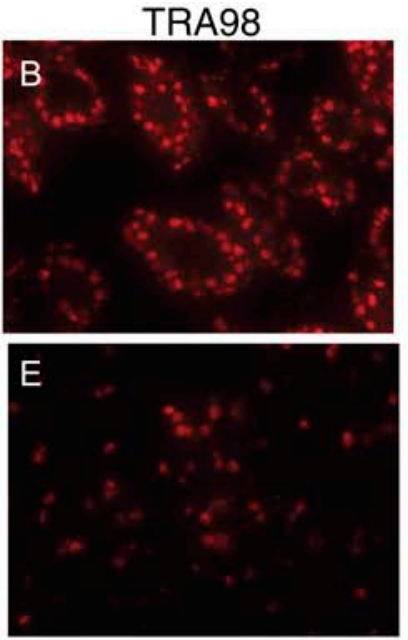

TRA98
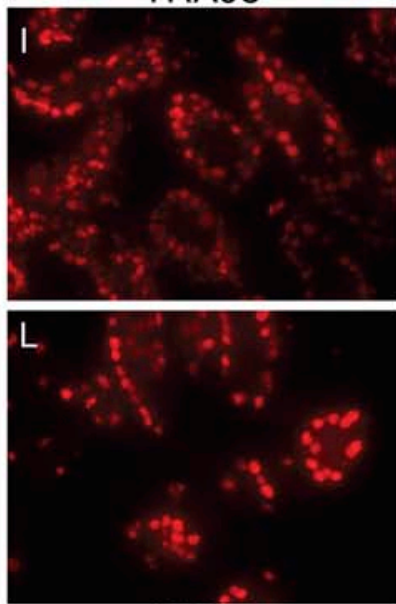

STRA8
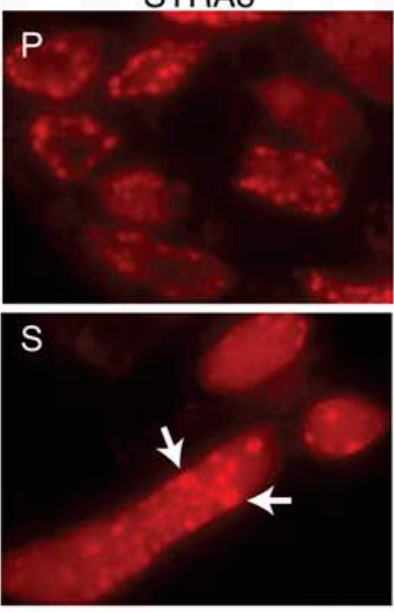
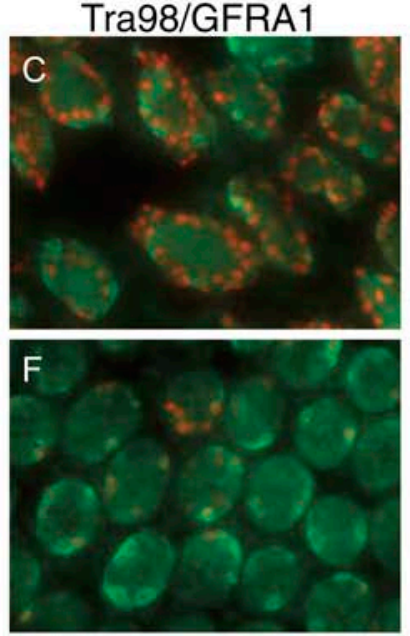

STRA8/TRA98
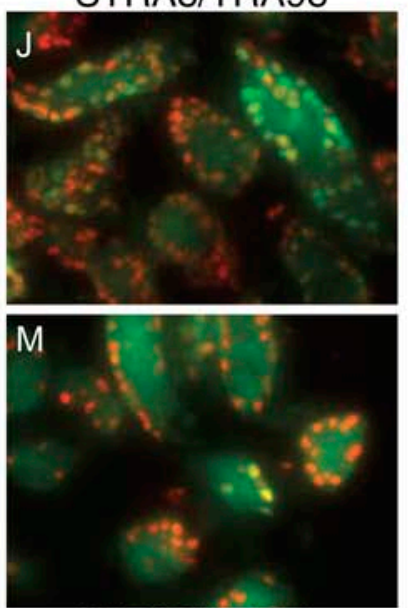

STRA8/GFRA1
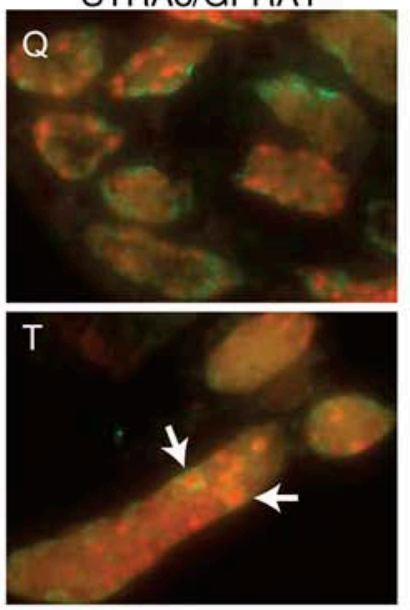

G

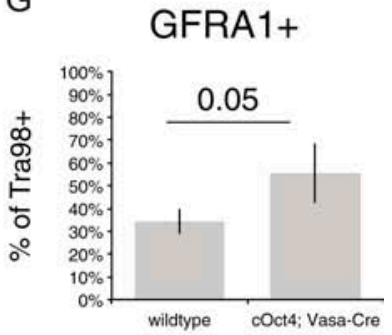

$\mathrm{N}$

STRA8+

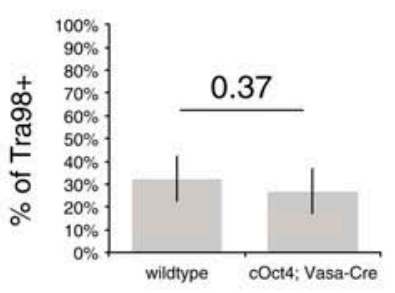

U

\section{GFRA1+/STRA8+ co-stained}

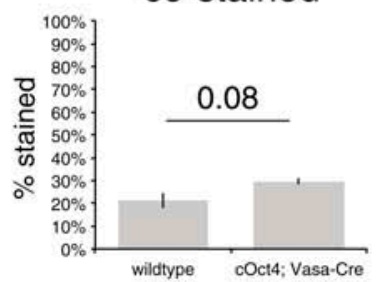

Figure 9 Fractions of undifferentiated and differentiated spermatogonia during first wave of spermatogenesis. (A, B, C, D, E and F) GFRA1 (green) and TRA98 (red) coimmunostaining to detect undifferentiated spermatogonia in wild type (A-C) and cOct4; Vasa-Cre (D-F). (G) Percentage of TRA98+ cells costained with GFRA1. Histogram shows mean and standard deviation for three wild-type mice (1130 TRA98+ cells) and three cOct4; Vasa-Cre mice (752 TRA98+ cells). (H, I, J, K, L and M) STRA8 (green) and TRA98 (red) coimmunostaining to detect differentiated spermatogonia in wildt ype $(\mathrm{H}-\mathrm{J})$ and cOct4; Vasa-Cre $(\mathrm{K}-\mathrm{M})$. (N) Percentage of TRA98+ cells costained with STRA8. Histogram shows mean and standard deviation for four wild-type mice (2627 TRA98+ cells) and five cOct4;Vasa-Cre mice (1711 TRA98+ cells). (O, P, Q, R, S and T) GFRA1 (green) and STRA8 (red) coimmunostaining in wild type (O-Q) and cOct4;Vasa-Cre (R-T). Arrows point to abnormally large nuclei. (U) Percentage of stained cells (either GFRA1+ or STRA8+) having costaining of GFRA1 and STRA8. Histogram shows mean and standard deviation for two wild-type mice (590 stained cells) and two cOct4; Vasa-Cre mice (403 stained cells). P-values are shown. Bar, $50 \mu \mathrm{m}$. 


\section{cOct4; Vasa-Cre leads to anomalies in spermatogonial survival, cell size and chain morphology}

Along with having fewer spermatogonia in cOct4; VasaCre neonatal mice, we noticed several other anomalies. We observed a slight increase in apoptosis at dpp 4 (Supplementary Fig. 5); however, the amount of apoptosis was unlikely to fully explain the dramatic reduction in germ cell numbers visible at dpp 6 . We also frequently observed abnormally large nuclei at dpp 6 , perhaps indicative of a cell cycle regulation defect that led to endoreduplication (Fig. 9R, S arrows and Supplementary Fig. 5). At dpp 13, cOct4; Vasa-Cre mice appeared to have an increase in the chain length and density of GFRA1+ cells, also suggestive of a defect in cell cycle regulation in the GFRA1+ population (Fig. 6B). Unusually long chains of GFRA1+ spermatogonia were also observed in adult mice (Fig. 6C-F). Altogether, these results suggest that spermatogonia in cOct4; Vasa-Cre proliferate abnormally and fail to appropriately make the transition to a differentiated state during the first wave of spermatogenesis.

\section{Discussion}

While POU5F1 is a well-known regulator of the pluripotent state, its function in the germ lineage has been less clear. Here, we focused on the postnatal expression and function of POU5F1. We found that POU51 expression in adults was downregulated compared with pups and that regulation of its levels in spermatogonia was proteosome dependent. Additionally, we showed that the regulation of POU5F1 expression was critical during the transition of gonocytes to differentiated spermatogonia and ultimately for the generation of meiotic germ cells. Substantial reductions in germ cells were observed by dpp 6, although interestingly the GFRA1+ undifferentiated spermatogonia population was largely spared. Consistent with the presence of selfrenewing stem cells, a population of undifferentiated spermatogonia was maintained into late adulthood (up to 12 months), and some of these SSCs escaped a differentiation block, leading to spermatogenic recovery in a few tubules. Nonetheless, a block in differentiation continued to be a major phenotype in adults, suggesting that the presence of ectopic POU5F1 during steadystate spermatogenesis had an inhibitory effect on differentiation, similar to its effect during the first wave of differentiation in neonates.

Using a variety of approaches, including quantitative comparisons of isolated cell populations, we showed that endogenous POU5F1 is detectable in both adult and neonatal undifferentiated spermatogonia. While we could detect low levels of POU5F1 in adult THY1+ cells by immunoblotting, and rare POU5F1+ cells in adults by two immunostaining approaches, POU5F1 was less abundant in adults compared with pups. Our data support the published data showing that POU5F1 is frequently but not exclusively present in $A_{s}$ and $A_{p r}$ cells and not observed in long chains of differentiated spermatogonia (Filipponi et al. 2007). In contrast, GFP expression in the widely used Oct4-GFP reporter mouse strain is present in differentiated spermatogonia in pups and adults. Hence, cells with endogenous POU5F1 are far less frequent than GFP+ cells in Oct4-GFP mice, which exhibit GFP in $A_{s}$ through $A_{\text {dif }}\left(A_{\text {differentiated }}\right)$ stages of spermatogonia (Ohbo et al. 2003, Garcia \& Hofmann 2012, Azizi et al. 2016). Immunoblotting of sorted cells confirmed that KIT+ differentiated spermatogonia express Oct4-GFP but not endogenous POU5F1 (Y Zheng and CT Dann, unpublished data). Altogether, the data support the notion that endogenous POU5F1 is present in a limited number of undifferentiated spermatogonia and that its overall expression diminishes over time from pup to adulthood.

The number of proteins like POU5F1 whose expression is limited to a subset of predominantly $A_{s}$ and $A_{p r}$ undifferentiated spermatogonia is growing and includes GFRA1, ID4 and PAX7 (Nakagawa et al. 2010, Oatley et al. 2011, Grasso et al. 2012, Aloisio et al. 2014). Whether individual cells expressing POU5F1 are the particular undifferentiated spermatogonia with stem cell activity is a challenging question. However, using transgenic reporter approaches and/or immunostaining and cell sorting, it was shown that populations of cells expressing Oct4-GFP or the other aforementioned proteins are enriched in stem cells (Ohbo et al. 2003, Ebata et al. 2005, Aloisio et al. 2014). Testing the relevance of each of these proteins for stem cell function is yet another question with significance but greatly difficult to answer because of the widespread roles each of these proteins plays during development.

Using an shRNA knockdown approach, we showed previously that Pou5f1 is required for SSC maintenance in vitro. In this study, we used an overexpression approach to test the function of POU5F1 in spermatogenesis in vivo. We used Stra8-Cre and Vasa-Cre drivers to remove the stop cassette from the transgene in the postnatal germ lineage, thereby forcing the expression of POU5F1 in all germ cells derived from cells having Cre-mediated excision. While Cre expression from the male germ cell-specific driver, Stra8-Cre, was originally reported to start at dpp 3 and continue through spermatocytes, recent reports suggest that excision may only begin to occur in slightly differentiated spermatogonia (Sadate-Ngatchou et al. 2008, Hobbs et al. 2015). Additionally, Bao and coworkers showed that incomplete excision occurs in the presence of two floxed alleles, but not one allele, leading to mosaicism (Bao et al. 2013). In cOct4;Stra8-Cre testes, in our study, we observed transgene expression (GFP) in spermatids in only a small subset of seminiferous tubule cross sections and there was no apparent phenotype. The reason for mosaic transgene expression in cOct4;Stra8-Cre testes 
may be a consequence of incomplete excision related to transgene copy number, variable Ubc promoter activity or weak GFP expression inherent in the use of T2A for producing two proteins from a single promoter. Still, the presence of some exogenous POU5F1 (detected by immunoblotting) combined with the GFP expression data suggest that the presence of ectopic POU5F1 in haploid spermatids in cOct4;Stra8-Cre mice may be inconsequential. In contrast, the testes of cOct4; VasaCre mice exhibited a striking phenotype. Our contrasting results when using the Stra8-Cre driver compared with the Vasa-Cre driver is similar to the experience recently reported by Hobbs and coworkers, who were also using the Stra8-Cre and Vasa-Cre drivers to study the effect of gene perturbation on spermatogonial stem/progenitor cells (Hobbs et al. 2015).

Vasa-Cre first leads to excision in males in the late embryonic germ cells and leads to 95\% efficient excision by birth (Gallardo et al. 2007). While the number and appearance of gonocytes in cOct4; Vasa-Cre mice at dpp 0 were normal, we could discern a modest decrease in the number of germ cells already at dpp 4 and a substantial reduction by dpp 6 . A key event that occurs during this stage is that some gonocytes develop into the first round of differentiated spermatogonia. Given that POU5F1 is normally present in undifferentiated spermatogonia, we predicted that forced expression of POU5F1 would not affect undifferentiated spermatogonia but instead may block differentiation. One possibility to explain the lower number of germ cells at dpp 6 following forced expression of POU5F1 is that the differentiation step is blocked. However, cOct4; Vasa-Cre mice still turned on the differentiation markers STRA8 (Fig. 9) and KIT (Y Zheng and CT Dann, unpublished data). We were unable to quantify a major shift in the portions of germ cells from a differentiated (STRA8+) to undifferentiated $($ GFRA $1+)$ state. The data may have been confounded by the presence of abnormal GFRA1+/STRA8+ spermatogonia, which could be categorized as neither undifferentiated nor differentiated. Nonetheless, the persistent expansion of GFRA1+ cells into adulthood and the continued presence of SALL4+ and GFRA1+ undifferentiated spermatogonia up to 12 months strongly imply that the stem cell population is not affected while major perturbations to differentiation prevail.

Another interesting aspect of the phenotype of cOct4; Vasa-Cre adults is that in some tubules, there is a significant spermatogenic recovery. This is particularly true in one of the three lines analyzed (line 21). We ruled out the possibility that 'leaky spermatogenesis' was a consequence of incomplete Cre excision (Supplementary Fig. 3 and data not shown) using PCR to show that sperm contained the excised version of the transgene. It is likely that the three lines represent an allelic series with higher (lines 53 and 18) and lower (line 21) expression of the transgene. A likely explanation for the leaky phenotype is that there are redundant mechanisms for regulating differentiation and/or downregulating POU5F1, and the amount of POU5F1 protein produced in cOct4;VasaCre germ cells was insufficient to lead to a complete differentiation block.

POU5F1 expression, regulation and function have been studied extensively in contexts such as ES cells and tumor cells. For instance, in ES cells, POU5F1 protein levels are ultimately determined by a combination of transcriptional control and posttranslational modifications including phosphorylation, sumoylation and ubiquitination (Ramakrishna et al. 2014). How POU5F1 levels are controlled in the postnatal and adult male germline remains unclear, but the presence of endogenous POU5F1 in fewer cells than the Oct4-GFP transcriptional reporter suggests that posttranscriptional and/or posttranslational mechanisms are likely to be involved in regulating POU5F1 during spermatogenesis. One explanation for the difficulty in detecting either endogenous or transgene-derived POU5F1 by immunostaining of testes may be related to the labile nature of the POU5F1 protein. Indeed, our data show that POU5F1 levels in cultured SSCs and testicular cells can be boosted by treatment with the proteosome inhibitor, MG132, similar to what has been shown in ES cells by others (Xu et al. 2004). POU5F1 may be ubiquitinated and quickly degraded in germ cells. Why POU5F1 could be detected by immunostaining in Oct4-GFP testes more readily than in other strains remains unclear. Further experimentation is necessary to overcome the issue of reliable POU5F1 detection by immunostaining and to dissect the pathways involved in regulating POU5F1 downregulation in spermatogenesis.

Another aspect of POU5F1 function that is of great interest is its potential role in tumorogenesis. POU5F1 is often aberrantly overexpressed in germ cell tumors, and in an ES cell-derived tumor model, the dose of POU5F1 was correlated with potential for malignancy (Gidekel et al. 2003). In a transgenic mouse model with a doxycycline-dependent expression system, ectopic POU5F1 led to dysplasia in epithelial tissues (Hochedlinger et al. 2005). However, in this model, it was not possible to address the role of ectopic POU5F1 in the germ lineage because doxycycline exposure did not result in POU5F1 overexpression in the testes, presumably because the blood-testes barrier prevented exposure of germ cells to doxycycline. Somewhat surprisingly, germ cell tumors were not observed in four cOct4;Vasa-Cre mice analyzed to date (10+ months); however, future studies may still be warranted (data not shown).

In summary, our results reveal the importance of POU5F1 regulation during neonatal and adult spermatogenesis. Just as POU5F1 levels must be strictly regulated during early embryogenesis for proper fate determination, the timing and levels of POU5F1 must be strictly controlled to allow the appropriate differentiation of SSCs into differentiated spermatogonia 
capable of undergoing meiosis. Finally, the creation of a transgenic mouse for conditional ectopic expression of POU5F1 may be a useful resource for studying the effect of POU5F1 regulation in other lineages during development or in pathological situations such as tumorogenesis or in studies of reprogramming to pluripotency.

\section{Supplementary data}

This is linked to the online version of the paper at http://dx.doi. org/10.1530/REP-16-0140.

\section{Declaration of interest}

The authors declare that there is no conflict of interest that could be perceived as prejudicing the impartiality of the research reported.

\section{Funding}

Research was supported by Eunice Kennedy Shriver National Institute of Child Health and Human Development of the National Institutes of Health under award number 4R01HD071081.

\section{Acknowledgements}

The authors thank Sue Childress, Aishwarya Narnur, Jennifer Balke and Angela Kerns for technical assistance, and Sachiko Koyama for critical reading of the manuscript. Indiana University Bloomington Light Microscopy Imaging Center and Flow Cytometery Core Facilities were used for certain experiments.

\section{References}

Aloisio GM, Nakada Y, Saatcioglu HD, Pena CG, Baker MD, Tarnawa ED, Mukherjee J, Manjunath H, Bugde A, Sengupta AL et al. 2014 PAX7 expression defines germline stem cells in the adult testis. Journal of Clinical Investigation 124 3929-3944. (doi:10.1172/JCI75943)

Azizi H, Conrad S, Hinz U, Asgari B, Nanus D, Peterziel H, Hajizadeh Moghaddam A, Baharvand H \& Skutella T 2016 Derivation of pluripotent cells from mouse SSCs seems to be age dependent. Stem Cells International 2016 8216312. (doi:10.1155/2016/8216312)

Bao J, Ma HY, Schuster A, Lin YM \& Yan W 2013 Incomplete cremediated excision leads to phenotypic differences between Stra8-iCre; Mov10l1(lox/lox) and Stra8-iCre; Mov10l1(lox/Delta) mice. Genesis 51 481-490. (doi:10.1002/dvg.22389)

Dann CT, Alvarado AL, Hammer RE \& Garbers DL 2006 Heritable and stable gene knockdown in rats. PNAS 103 11246-11251. (doi:10.1073/ pnas.0604657103)

Dann CT, Alvarado AL, Molyneux LA, Denard BS, Garbers DL \& Porteus MH 2008 Spermatogonial stem cell self-renewal requires OCT4, a factor downregulated during retinoic acid-induced differentiation. Stem Cells 26 2928-2937. (doi:10.1634/stemcells.2008-0134)

Ebata KT, Zhang X \& Nagano MC 2005 Expression patterns of cellsurface molecules on male germ line stem cells during postnatal mouse development. Molecular Reproduction and Development 72 171-181. (doi:10.1002/(ISSN)1098-2795)
Fanslow DA, Wirt SE, Barker JC, Connelly JP, Porteus MH \& Dann CT 2014 Genome editing in mouse spermatogonial stem/progenitor cells using engineered nucleases. PLOS ONE 9 e112652. (doi:10.1371/journal. pone.0112652)

Filipponi D, Hobbs RM, Ottolenghi S, Rossi P, Jannini EA, Pandolfi PP \& Dolci S 2007 Repression of kit expression by Plzf in germ cells. Molecular and Cellular Biology 27 6770-6781. (doi:10.1128/MCB.00479-07)

Foulkes NS, Mellstrom B, Benusiglio E \& Sassone-Corsi P 1992 Developmental switch of CREM function during spermatogenesis: from antagonist to activator. Nature 355 80-84. (doi:10.1038/355080a0)

Gallardo T, Shirley L, John GB \& Castrillon DH 2007 Generation of a germ cell-specific mouse transgenic Cre line, Vasa-Cre. Genesis 45 413-417. (doi:10.1002/(ISSN)1526-968X)

Garcia T \& Hofmann MC 2012 Isolation of undifferentiated and early differentiating type A spermatogonia from Pou5f1-GFP reporter mice. Methods in Molecular Biology 825 31-44. (doi:10.1007/978-1-61779436-0)

Gassei K \& Orwig KE 2013 SALL4 expression in gonocytes and spermatogonial clones of postnatal mouse testes. PLOS ONE 8 e53976. (doi:10.1371/journal.pone.0053976)

Gibson DG, Glass JI, Lartigue C, Noskov VN, Chuang RY, Algire MA, Benders GA, Montague MG, Ma L, Moodie MM et al. 2010a Creation of a bacterial cell controlled by a chemically synthesized genome. Science 329 52-56. (doi:10.1126/science.1190719)

Gibson DG, Smith HO, Hutchison CA 3rd, Venter JC \& Merryman C $2010 b$ Chemical synthesis of the mouse mitochondrial genome. Nature Methods 7 901-903. (doi:10.1038/nmeth.1515)

Gidekel S, Pizov G, Bergman Y \& Pikarsky E 2003 Oct-3/4 is a dosedependent oncogenic fate determinant. Cancer Cell 4 361-370. (doi:10.1016/S1535-6108(03)00270-8)

Grasso M, Fuso A, Dovere L, de Rooij DG, Stefanini M, Boitani C \& Vicini E 2012 Distribution of GFRA1-expressing spermatogonia in adult mouse testis. Reproduction 143 325-332. (doi:10.1530/REP-11-0385)

Griffin J 2013 Methods of sperm DNA extraction for genetic and epigenetic studies. Methods in Molecular Biology 927 379-384. (doi:10.1007/9781-62703-038-0)

Gu G, Dubauskaite J \& Melton DA 2002 Direct evidence for the pancreatic lineage: NGN3+ cells are islet progenitors and are distinct from duct progenitors. Development 129 2447-2457.

Heim C, Minniear K \& Dann CT 2011 Imatinib has deleterious effects on differentiating spermatogonia while sparing spermatogonial stem cell self renewal. Reproductive Toxicology 31 454-463. (doi:10.1016/j. reprotox.2010.12.056)

Hobbs RM, La HM, Makela JA, Kobayashi T, Noda T \& Pandolfi PP 2015 Distinct germline progenitor subsets defined through Tsc2-mTORC1 signaling. EMBO Reports 16 467-480. (doi:10.15252/embr.201439379)

Hochedlinger K, Yamada Y, Beard C \& Jaenisch R 2005 Ectopic expression of Oct-4 blocks progenitor-cell differentiation and causes dysplasia in epithelial tissues. Cell 121 465-477. (doi:10.1016/j.cell.2005.02.018)

Hofmann MC, Braydich-Stolle L \& Dym M 2005 Isolation of male germ-line stem cells; influence of GDNF. Developmental Biology 279 114-124. (doi:10.1016/j.ydbio.2004.12.006)

Kanatsu-Shinohara M, Ogonuki N, Inoue K, Miki H, Ogura A, Toyokuni S \& Shinohara T 2003 Long-term proliferation in culture and germline transmission of mouse male germline stem cells. Biology of Reproduction 69 612-616. (doi:10.1095/biolreprod.103.017012)

Kehler J, Tolkunova E, Koschorz B, Pesce M, Gentile L, Boiani M, Lomeli H, Nagy A, McLaughlin KJ, Scholer HR et al. 2004 Oct4 is required for primordial germ cell survival. EMBO Reports 5 1078-1083. (doi:10.1038/sj.embor.7400279)

Ketkar AA \& Reddy KVR 2012 Expression pattern of OCT4 and Plzf transcription factors during the early events of spermatogenesis in mice. Cell Science and Therapy 3. (doi:10.4172/2157-7013.1000120)

Madisen L, Zwingman TA, Sunkin SM, Oh SW, Zariwala HA, Gu H, Ng LL, Palmiter RD, Hawrylycz MJ, Jones AR et al. 2010 A robust and high-throughput Cre reporting and characterization system for the whole mouse brain. Nature Neuroscience 13 133-140. (doi:10.1038/ nn.2467)

Nakagawa T, Sharma M, Nabeshima Y, Braun RE \& Yoshida S 2010 Functional hierarchy and reversibility within the murine spermatogenic stem cell compartment. Science 328 62-67. (doi:10.1126/science.1182868) 
Nichols J, Zevnik B, Anastassiadis K, Niwa H, Klewe-Nebenius D, Chambers I, Scholer H \& Smith A 1998 Formation of pluripotent stem cells in the mammalian embryo depends on the POU transcription factor Oct4. Cell 95 379-391. (doi:10.1016/S0092-8674(00)81769-9)

Niedenberger BA, Busada JT \& Geyer CB 2015 Marker expression reveals heterogeneity of spermatogonia in the neonatal mouse testis. Reproduction 149 329-338. (doi:10.1530/REP-14-0653)

Niwa H, Miyazaki J \& Smith AG 2000 Quantitative expression of Oct3/4 defines differentiation, dedifferentiation or self-renewal of ES cells. Nature Genetics 24 372-376. (doi:10.1038/74199)

Oatley MJ, Kaucher AV, Racicot KE \& Oatley JM 2011 Inhibitor of DNA binding 4 is expressed selectively by single spermatogonia in the male germline and regulates the self-renewal of spermatogonial stem cells in mice. Biology of Reproduction 85 347-356. (doi:10.1095/ biolreprod.111.091330)

Ohbo K, Yoshida S, Ohmura M, Ohneda O, Ogawa T, Tsuchiya H, Kuwana T, Kehler J, Abe K, Scholer HR et al. 2003 Identification and characterization of stem cells in prepubertal spermatogenesis in mice. Developmental Biology 258 209-225. (doi:10.1016/S00121606(03)00111-8)

Palmieri SL, Peter W, Hess H \& Scholer HR 1994 Oct-4 transcription factor is differentially expressed in the mouse embryo during establishment of the first two extraembryonic cell lineages involved in implantation. Developmental Biology 166 259-267. (doi:10.1006/dbio.1994.1312)

Pesce M, Wang X, Wolgemuth DJ \& Scholer H 1998 Differential expression of the Oct-4 transcription factor during mouse germ cell differentiation. Mechanisms of Development 71 89-98. (doi:10.1016/S0925 4773(98)00002-1)

Ramakrishna S, Kim KS \& Baek KH 2014 Posttranslational modifications of defined embryonic reprogramming transcription factors. Cellular Reprogramming 16 108-120. (doi:10.1089/cell.2013.0077)

Rosner MH, Vigano MA, Ozato K, Timmons PM, Poirier F, Rigby PW \& Staudt LM 1990 A POU-domain transcription factor in early stem cells and germ cells of the mammalian embryo. Nature 345 686-692. (doi:10.1038/345686a0)

Sadate-Ngatchou PI, Payne CJ, Dearth AT \& Braun RE 2008 Cre recombinase activity specific to postnatal, premeiotic male germ cells in transgenic mice. Genesis 46 738-742. (doi:10.1002/dvg.v46:12)

Scholer HR, Dressler GR, Balling R, Rohdewohld H \& Gruss P 1990a Oct-4: a germline-specific transcription factor mapping to the mouse t-complex. EMBO Journal 9 2185-2195.

Scholer HR, Ruppert S, Suzuki N, Chowdhury K \& Gruss P 1990 b New type of POU domain in germ line-specific protein Oct-4. Nature 344 435-439. (doi:10.1038/344435a0)

Schorpp M, Jager R, Schellander K, Schenkel J, Wagner EF, Weiher H \& Angel P 1996 The human ubiquitin C promoter directs high ubiquitous expression of transgenes in mice. Nucleic Acids Research $\mathbf{2 4}$ 1787-1788. (doi:10.1093/nar/24.9.1787)

Schrans-Stassen BH, van de Kant HJ, de Rooij DG \& van Pelt AM 1999 Differential expression of c-kit in mouse undifferentiated and differentiating type A spermatogonia. Endocrinology 140 5894-5900. (doi:10.1210/endo.140.12.7172)
Szabo PE, Hubner K, Scholer H \& Mann JR 2002 Allele-specific expression of imprinted genes in mouse migratory primordial germ cells. Mechanisms of Development 115 157-160. (doi:10.1016/S09254773(02)00087-4)

Tadokoro Y, Yomogida K, Ohta H, Tohda A \& Nishimune Y 2002 Homeostatic regulation of germinal stem cell proliferation by the GDNF/ FSH pathway. Mechanisms of Development 113 29-39. (doi:10.1016/ S0925-4773(02)00004-7)

Tanaka H, Pereira LA, Nozaki M, Tsuchida J, Sawada K, Mori H \& Nishimune Y 1997 A germ cell-specific nuclear antigen recognized by a monoclonal antibody raised against mouse testicular germ cells. International Journal of Andrology 20 361-366. (doi:10.1046/j.13652605.1998.00080.x)

Tokuda M, Kadokawa Y, Kurahashi H \& Marunouchi T 2007 CDH1 is a specific marker for undifferentiated spermatogonia in mouse testes. Biology of Reproduction 76 130-141. (doi:10.1095/ biolreprod.106.053181)

Wu X, Oatley JM, Oatley MJ, Kaucher AV, Avarbock MR \& Brinster RL 2010 The POU domain transcription factor POU3F1 is an important intrinsic regulator of GDNF-induced survival and self-renewal of mouse spermatogonial stem cells. Biology of Reproduction 82 1103-1111. (doi:10.1095/biolreprod.109.083097)

Xu HM, Liao B, Zhang QJ, Wang BB, Li H, Zhong XM, Sheng HZ, Zhao YX, Zhao YM \& Jin Y 2004 Wwp2, an E3 ubiquitin ligase that targets transcription factor Oct-4 for ubiquitination. Journal of Biological Chemistry 279 23495-23503. (doi:10.1074/jbc.M400516200)

Yang S, Cohen CJ, Peng PD, Zhao Y, Cassard L, Yu Z, Zheng Z, Jones S, Restifo NP, Rosenberg SA et al. 2008 Development of optimal bicistronic lentiviral vectors facilitates high-level TCR gene expression and robust tumor cell recognition. Gene Therapy 15 1411-1423. (doi:10.1038/ gt.2008.90)

Yeom YI, Fuhrmann G, Ovitt CE, Brehm A, Ohbo K, Gross M, Hubner K \& Scholer HR 1996 Germline regulatory element of Oct-4 specific for the totipotent cycle of embryonal cells. Development 122 881-894.

Yoshida S, Sukeno M, Nakagawa T, Ohbo K, Nagamatsu G, Suda T \& Nabeshima Y 2006 The first round of mouse spermatogenesis is a distinctive program that lacks the self-renewing spermatogonia stage. Development 133 1495-1505. (doi:10.1242/dev.02316)

Zhou Q, Nie R, Li Y, Friel P, Mitchell D, Hess RA, Small C \& Griswold MD 2008 Expression of stimulated by retinoic acid gene 8 (Stra8) in spermatogenic cells induced by retinoic acid: an in vivo study in vitamin A-sufficient postnatal murine testes. Biology of Reproduction 79 35-42. (doi:10.1095/biolreprod.107.066795)

Received 15 March 2016

First decision 21 April 2016

Revised manuscript received 23 June 2016

Accepted 1 August 2016 